\title{
Axion production from gravitons off interacting 0-branes
}

\author{
Faheem Hussain ${ }^{1}$, Roberto Iengo ${ }^{2}$ and Carmen Núñez ${ }^{3}$ \\ ${ }^{1}$ International Centre for Theoretical Physics, Trieste, Italy \\ 2 International School for Advanced Studies, Trieste, Italy \\ ${ }^{3}$ Instituto de Astronomía y Física del Espacio (CONICET), Buenos Aires, Argentina
}

\begin{abstract}
We study axion-graviton scattering from a system of two D0-branes in a Type II superstring theory, a process which does not occur on a single brane. The two D0-branes interact via the exchange of closed string states which form a cylinder joining them. By compactifying on the $Z_{3}$ orbifold we find a non vanishing amplitude coming from the odd spin structure sector, thus from the exchanged RR states. We compute, in particular, the leading term of the amplitude at large distance from the branes, which corresponds to taking a field theory limit. This seems to suggest that the process takes place through the coupling of an axion to the RR states exchanged between the 0-branes.
\end{abstract}

PACS: 11.25.-w

Keywords: string theory, D-branes 


\section{INTRODUCTION}

String duality, a symmetry of string theory, gives information about the behavior of string theory at strong coupling. It provides evidence about nonperturbative aspects of the theory. This symmetry implies that the strongly coupled limit of every string theory is equivalent to the weakly coupled limit of some other theory. It is now believed that the known superstring theories are different realisations of some more fundamental underlying theory. In order to understand the nature of this unknown theory it seems necessary to gather as much data about it as possible by studying examples of stringy vacua.

Most non-perturbative string dualities require the existence of elementary RamondRamond (RR) charges which couple to the RR fields contained in the spectrum of type-I and type-II superstring theories [1,2] As is well known string perturbation theory contains no such elementary RR charges. Since a $(p+1)$ form couples naturally to a $p$-brane, an extended object with $p$ spatial dimensions, duality requires us to consider such branes carrying RR charges. Such $p$ - branes were originally found and studied as soliton solutions of the effective low-energy supergravity [3]. However, following Polchinski's work [4] it has become clear that there is a much simpler description of $p$-branes carrying RR charges. This description amounts to considering $p$-branes to which end points of open strings can be attached. This is accomplished by imposing Dirichlet boundary conditions on the world sheet fields, hence the name $\mathrm{D} p$-branes or D-branes.

Since string theory contains gravity, the D-branes are also a source for the gravitational field and therefore they represent a class of "black" objects of the family of generalized black holes. Actually, the RR charged solitons required by string duality first appeared as black $p$-brane solutions to the low energy effective theory. The conformal field theory description of D-branes proposed in [⿴囗十] has allowed considerable progress in accounting for the black hole information paradox and in probing the nature of spacetime at the shortest distance scales. Examples of black holes in four and five dimensions have been constructed for which the degeneracy of microscopic D-brane states matches the Bekenstein-Hawking entropy [5]. 
This conformal field theory formulation consists of type II superstrings with mixed Dirichlet and Neumann boundary conditions. Such a world sheet approach has made it possible to explicitly compute several properties which had been anticipated by arguments of duality and supersymmetry. One of the most intriguing hints from duality is the appearance of new length scales in string theory [6]. By studying zero-brane dynamics it was possible to probe distances much shorter than the string scale [7]. Scattering of massless closed string states off D-branes at leading order in perturbation theory have made manifest the stringy features of the RR solitons [8,9], such as the Regge behaviour and the exponential fall off of fixed angle scattering [10. The exchange of massless states on the disk at zero momentum transfer has been shown to agree with the $p$-brane solution to the effective field theory at low energies. A systematic approach to all massless two point functions on a disk was recently developed in [11,12] where it was shown that there is a direct relation between four point functions of type I theory and two point functions of type II theory in a D-brane background.

In this paper we study the axion production amplitude from an incoming graviton off a system of two D0-branes, or D-particles, in a Superstring Type II theory. Actually, we will see that both choices of Neumann or Dirichlet boundary conditions in the compactified coordinates give the same result, and thus we speak of 0-branes referring to the uncompactified coordinates.

The amplitude for this process vanishes in the case of one D-brane [11]. Therefore, the brane-brane interaction plays a crucial role in our interesting non trivial result and the process can be regarded as a mechanism to obtain novel features of the physics of D-branes. The two 0-branes interact via the exchange of closed string states which form a cylinder joining them. Computing the above amplitude implies the insertion of two NS-NS vertices on the cylinder with the appropriate boundary conditions. It is easy to show that this leads to a vanishing result in 10 dimensions because of the abstruse identity. Having in mind a more interesting scenario, we consider a realistic compactification scheme breaking the supersymmetry down to $N=2$ in 4 dimensions. The presence of the branes will further reduce the supersymmetry to $N=1$. In order to be able to do an explicit computation we 
consider compactification on the standard $Z_{3}$ orbifold. This breaks enough supersymmetry to allow a non-zero result.

We find that the only non-zero contribution to the particular axion-graviton amplitude under consideration, comes from the odd spin structure sector (recall that the boundary conditions for world sheet fermions can be classified according to the spin-structure). Interestingly enough, the odd spin-structure corresponds to one term of the GSO projection of the RR world sheet fermions. We also find that the only contribution to the odd spin structure amplitude from the compactified coordinates arises in the twisted sector of the closed string (recall that in an orbifold compactification twisted and untwisted sectors, by an element of the symmetry group, have to be considered).

The branes cannot transfer energy but they can transfer momenta. Poles in the momentum transfer arise when the vertices corresponding to the graviton and the axion come together on the cylinder. We refer to this process as the pinching limit. This pole signals the propagation of a massless closed string state, which couples to the the point where the axion and graviton vertices come together. It could be interpreted as a virtual axion, propagating out of the two branes' system, which is eventually made real by absorbing the incoming graviton. The residue of the pole can also be singular rather than constant. This further singularity signals the propagation of massless closed string states between the branes, whose proper time is the length of the cylinder. The region in which the length of the cylinder diverges can be viewed as an infrared limit of long time propagation, the field theory limit. It corresponds to the exchange of the lowest closed string states, showing the suppression of massive closed string states at large distances. We study the amplitude in the pinching limit and field theory limit, taking together all the possible sources of singularities in order to find the leading behavior for small momentum transfer, corresponding to large distances. The structure of the resulting expression in this double limit seems to suggest that the process takes place through the exchange of an axion which couples to the RR states being exchanged between the D-particles.

We can also think of our amplitude containing two 0-branes as the second order pertur- 
bative term in the classical (i.e. tree level) evaluation of the graviton-axion scattering off a single 0-brane. Namely, in this picture the 0-brane plays the role of a source, and the classical perturbative expansion of a nonlinear field theory with a source gives rise to terms in each of which the source appears repeatedly [13]. We will be interested in the evaluation of the amplitude for small momentum transfer, that is at large distances from the source. Thus we could interpret the intermediate states coming out of the branes as the halo of the fields surrounding the gravitational object represented by the 0-brane. In particular, in this paper we compute a scattering amplitude which is zero on one 0-brane [1], that is at the lowest classical perturbative order, and thus we can interpret the result as an effect due to the halo. Our computation gives the exact leading result for large distances.

We have organized the paper such that the arguments can be followed without being distracted by too much side information or computations and therefore we have kept in the main text only what is strictly necessary for its understanding. Additional information and technical details have been put in appendices. In Section $\mathbb{1}$ we compute the correlators of bosonic and fermionic world sheet fields on the cylinder. In Section III we present some general considerations on how to construct the scattering amplitude for two NS-NS fields using the closed string formulation. In this section we also show how the ghosts are consistently taken care of, so that we can subsequently work in the light cone formalism. In Section IV we review the construction for the RR boundary state and show that the compactified untwisted sector of the closed string does not contribute to the amplitude. In Section $\square$ we calculate the final form of the scattering amplitude for the graviton-axion off the two 0-branes. In Section $\nabla 1$ we consider the pinching limit and the behaviour of the amplitude at small $q^{2}$ and discuss the result. The content of the Appendices is the following: in Appendix $\mathrm{A}$ and $\mathrm{B}$ we describe the vacuum amplitude (that is the case without vertex insertions) for the two interacting branes, first reviewing the $\mathrm{N}=4$ supersymmetric case in App.A and then constructing the orbifold case giving $\mathrm{N}=1$ in App.B. In Appendix $\mathrm{C}$ we show the vanishing of the even spin structures' contribution, and in Appendix D we complete the analysis of the field theory limit by computing subleading terms. 


\section{CORRELATORS ON THE CYLINDER}

In this section we review the construction of the cylinder and the correlators of bosonic and fermionic fields on the cylinder.

D-branes are described by open superstrings with their end points fixed on a $(p+1)$ dimensional hypersurface embedded in ten dimensional spacetime. The coordinates $X^{A}$, $(A=0, \ldots, p)$ with conventional Neumann boundary conditions, span the world volume swept by the $p$-brane, whereas $X^{i},(i=p+1, \ldots, 9)$ label the transverse directions with Dirichlet boundary conditions.

Let us consider a cylinder joining two branes at $X^{i}\left(\sigma_{1}=0, \sigma_{2}\right)=0$ and $X^{i}\left(\sigma_{1}=l, \sigma_{2}\right)=Y^{i}$, as shown in Figure 1. This can be interpreted as a closed string state appearing from one D-brane, propagating in Euclidean time $i \sigma_{1}=l$ and disappearing in the other [四]. $\sigma_{2}$ is a periodic coordinate running from 0 to 1 .

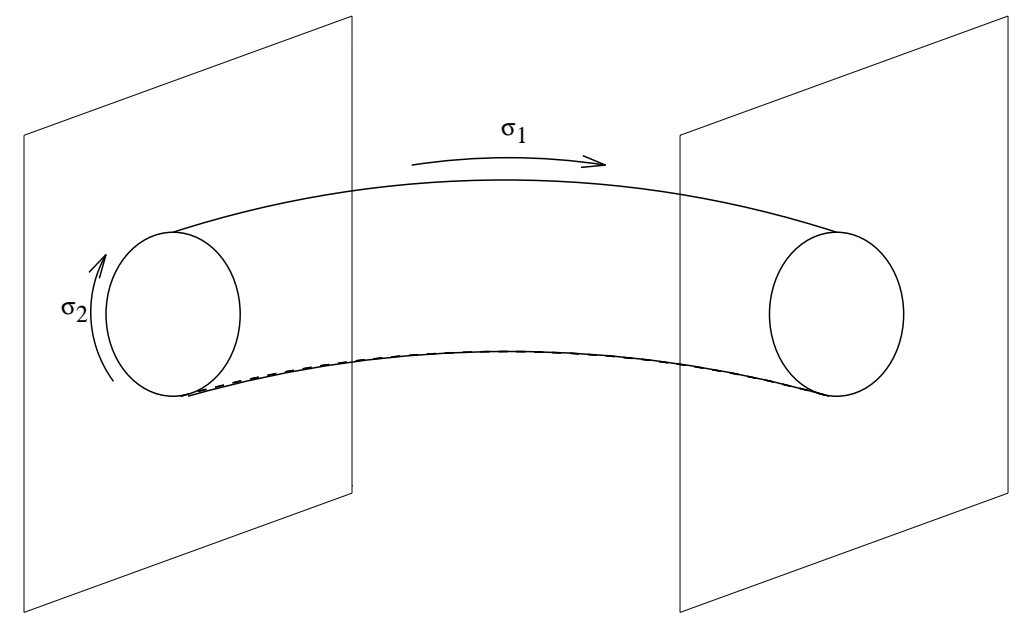

Figure 1

We map the cylinder joining the two branes into a world sheet cylinder. Following Burgess and Morris [14] we construct this cylinder from the torus. Using the complex coordinate $w=\sigma_{2}+i \sigma_{1}$, the torus in Figure 2 is the rectangle $0 \leq \sigma_{1} \leq \operatorname{Im} \tau, 0 \leq \sigma_{2} \leq 1$. The cylinder is obtained by reflecting about the dotted line $\sigma_{1}=\operatorname{Im} \tau / 2$ and identifying the segments OA with BC. This corresponds to the involution $w \rightarrow \bar{w}+i \tau$ and leaves invariant $\sigma_{1}=0$ and $\sigma_{1}=\tau / 2$. Note that we have taken $\tau=i \nu$ pure imaginary, and $\nu=2 l$ is the real 
Teichmuller parameter in terms of the length of the cylinder.

In these coordinates the Neumann boundary conditions for the bosonic fields are

$$
\partial_{\sigma_{1}} X^{A}\left(\sigma_{1}=0, \sigma_{2}\right)=\partial_{\sigma_{1}} X^{A}\left(\sigma_{1}=l, \sigma_{2}\right)=0,
$$

whereas the Dirichlet boundary conditions, $X^{i}\left(\sigma_{1}=0, \sigma^{2}\right)=0, X^{i}\left(\sigma_{1}=l, \sigma^{2}\right)=Y^{i}$ are implemented by writing

$$
X^{i}(w)=\frac{Y^{i} I m w}{l}+X_{q u a n t u m}^{i}(w)
$$

with

$$
X_{\text {quantum }}^{i}\left(\sigma_{1}=0\right)=X_{\text {quantum }}^{i}\left(\sigma_{1}=l\right)=0,
$$

and $Y^{i}$ is the separation of the D-branes in the $i$ direction.

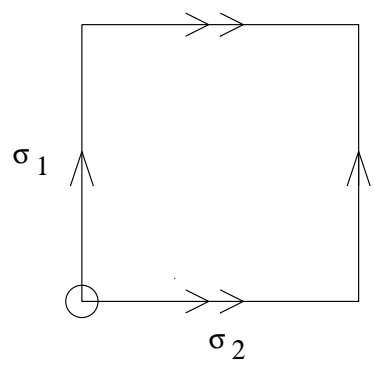

TORUS

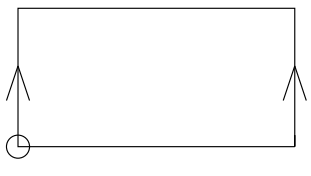

CYLINDER

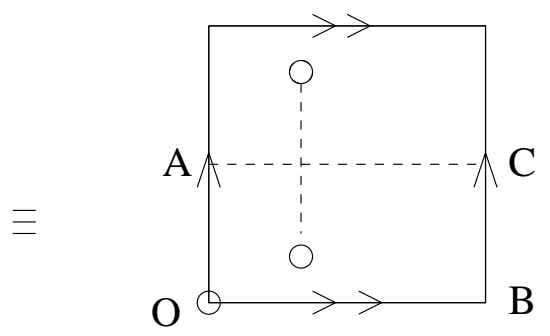

Figure 2

With these boundary conditions, we can then construct the bosonic and fermionic propagators on the cylinder using the usual torus propagators. For fermions, the operator representing the involution $\sigma_{1} \rightarrow 1-\sigma_{1}$ is $\gamma_{1}$ [14 in the two dimensional spinor space. It follows from world sheet supersymmetry that the Neumann boundary conditions are 
$\Psi^{A}(\bar{w}+2 i l)=\gamma_{1} \Psi^{A}(w)$, where $\Psi=\left[\begin{array}{l}\psi(w) \\ \bar{\psi}(\bar{w})\end{array}\right]$, giving $\bar{\psi}^{A}(\bar{w})=\psi^{A}(\bar{w}+2 i l)$, whereas for Dirichlet boundary conditions we get $\bar{\psi}^{i}(\bar{w})=-\psi^{i}(\bar{w}+2 i l)$. Here and subsequently $\psi(\bar{\psi})$ mean right (left) moving fields respectively. We can drop the shift $2 i l$ from the argument, which just brings us back to the upper half plane into the torus. In other words instead of working in the upper half plane we can equally well work in the strip $-i l<\operatorname{Im} z<i l, 0<\operatorname{Re} z<1$. Also in this paper we are only concerned with D0-branes in the uncompactified directions. The vertices that we need to insert on the cylinder contain only uncompactified string coordinates. Thus we can restrict here the discussion to the case in which the coordinate index is $\mu=0,1,2,3$. The coordinates and the fermions in the time direction have Neumann boundary conditions, whereas the ones in the three space directions have Dirichlet conditions. Thus the boundary conditions on the fermions can be written in a compact form as

$$
\bar{\psi}^{\mu}(\bar{z})=S_{\nu}^{\mu} \psi^{\nu}(x)
$$

with $x=\bar{z}$ and $S_{n}^{m}=-\delta_{n}^{m}, S_{0}^{0}=1$ and off diagonal $=0$. Thus we only need to specify the $\left\langle\psi\left(x_{1}\right) \psi\left(x_{2}\right)\right\rangle$ correlator, $x_{1}, x_{2}$ being complex coordinates.

The above notation can be used for the correlators of the bosonic coordinates, which can be written as

$$
<X^{\mu}(z, \bar{z}) X^{\nu}(w, \bar{w})>=-\eta^{\mu \nu} G(z, w)-S^{\mu \nu} G(z, \bar{w})
$$

where

$$
G\left(x_{1}, x_{2}\right)=\ln \left|\frac{\vartheta_{1}\left(x_{1}-x_{2} \mid \tau\right)}{\vartheta_{1}^{\prime}(0)}\right|^{2}-2 \pi \frac{\left(\operatorname{Im}\left(x_{1}-x_{2}\right)\right)^{2}}{\operatorname{Im} \tau}
$$

and $\vartheta_{1}\left(x_{1}-x_{2} \mid \tau\right)=\vartheta\left[\begin{array}{l}1 / 2 \\ 1 / 2\end{array}\right]\left(x_{1}-x_{2} \mid \tau\right)$.

For the fermionic correlators one has to specify the spin structure $s$. For the three even spin structures we have

$$
<\psi^{\mu}\left(x_{1}\right) \psi^{\nu}\left(x_{2}\right)>_{s}=-\eta^{\mu \nu} \frac{\vartheta_{s}\left(x_{1}-x_{2} \mid \tau\right)}{\vartheta_{1}\left(x_{1}-x_{2} \mid \tau\right)} \frac{\vartheta_{1}^{\prime}(0)}{\vartheta_{s}(0)}=-\eta^{\mu \nu} F_{s}\left(x_{1}-x_{2}\right)
$$


where $\vartheta_{s}\left(x_{1}-x_{2} \mid \tau\right), s=2,3,4$ are defined in Appendix A. As for the $\left\langle\psi \psi>_{\text {odd }}\right.$ correlators, they are determined by the requirement that they have one pole at $1 /\left(x_{1}-x_{2}\right)$, like $\partial_{x_{1}} G\left(x_{1}, x_{2}\right)$. In fact, since in the odd spin structure case $\psi$ has the same periodicity conditions as $X$, these requirements give

$$
<\psi^{\mu}(z) \psi^{\nu}(w)>_{o d d}=-\eta^{\mu \nu} \partial_{x_{1}} G\left(x_{1}, x_{2}\right)=-\eta^{\mu \nu} F\left(x_{1}-x_{2}\right)
$$

Remember that, due to eq.(2.4), eqs.(2.7) and (2.8) also encode the $\langle\psi \bar{\psi}\rangle$ and $\langle\bar{\psi} \bar{\psi}\rangle$ propagators. Actually the odd case requires a more complete discussion. We come back to that at the end of the next section.

\section{CONSTRUCTION OF SCATTERING AMPLITUDE}

As mentioned in the introduction we consider the scattering of NS-NS fields from a system of two 0-branes in a superstring theory compactified down to four dimensions on the standard $Z_{3}$ orbifold. The cylinder depicted in Figure 1 is a one loop open string graph. The world line of the open string boundary can be regarded as a state connecting the vacuum to one closed string. Thus one has to first construct boundary states as we do our calculation in the closed string formulation. Our 0-branes are such that only the time coordinate $X^{0}$ has Neumann boundary conditions. The three uncompactified space coordinates have Dirichlet boundary conditions. Using the conventions of ref. [15], the Neumann and Dirichlet boundary conditions translate into the following conditions for the boundary state $(n$ means modes of the $\sigma_{2}$ Fourier expansion):

$$
\left(\alpha_{n}^{0}+\tilde{\alpha}_{-n}^{0}\right)\left|B>=0, \quad\left(\psi_{n}^{0}+i \eta \tilde{\psi}_{-n}^{0}\right)\right| B>=0
$$

and

$$
\left(\alpha_{n}^{i}-\tilde{\alpha}_{-n}^{i}\right)\left|B>=0, \quad\left(\psi_{n}^{i}-i \eta \tilde{\psi}_{-n}^{i}\right)\right| B>=0
$$

for all $n$. Here $i=1,2,3$ denote the three uncompactified space directions and the tilded or untilded operators correspond to the mode expansion of right and left movers. Further, 
$\eta= \pm 1$ and we will see that the different spin structures arise from taking the same or opposite value of $\eta$ for the boundary states of the two branes respectively.

Here we have lumped together NS-NS and R-R sectors. These are specified by taking $n$ to be half-integer or integer, repectively in the mode expansion for the fermions. To be general we also have to specify the boundary state conditions for the six compact coordinates, $X^{4} \cdots X^{9}$. One has to keep several things in mind here. The boundary state condition will include not only the spin structure factor $\eta$ and Neumann (Dirichlet) signs but one has also to project onto the $Z_{3}$ invariant states [16]. We shall discuss this further later in this section. Also we have to remember that in the $\sigma_{2}$ twisted sectors the moding is not integral or half integral but in integer multiples of $1 / 3$. These conditions implicitly define the boundary state $\mid B, k>$, with momentum $k$.

Then the boundary states on the two 0-branes are given by

$$
\begin{aligned}
\mid B, X^{i}=0> & =\int \frac{d^{3} k}{(2 \pi)^{3}} e^{i k \cdot(X=0)} \mid B, k> \\
\mid B, X^{i}=Y^{i}> & =\int \frac{d^{3} k}{(2 \pi)^{3}} e^{i k \cdot Y} \mid B, k>
\end{aligned}
$$

Now we review the general construction of the scattering amplitude, leaving the details to be explained in other Sections. The scattering amplitude $A$ can be written as a path integral over world-sheet fields on a cylinder. The integrand is the product of two NS-NS vertices, $V$ and $U$, in the 0 -ghost picture, constructed from world sheet fields with components only in the uncompactified directions (remember that on the cylinder the worldsheet left and right moving fields are identified up to some phase). The result of the integration can be cast in the form

$$
A(l ; z, w)=\sum_{s}( \pm)<U(z, \bar{z}) V(w, \bar{w})>_{s} \cdot Z_{s},
$$

where $\langle U V\rangle_{s}$ is evaluated in terms of correlators by using the Wick theorem, and $Z_{s}$ is the socalled partition function given by

$$
Z_{s}=<B\left|e^{-l H_{s}}\right| B>_{s},
$$


where $H_{s}$ is the relevant Hamiltonian for the world sheet fields. For the fermionic coordinates one has to specify the spin structure $s$ and sum over the spin structures with appropriate signs as indicated above. The four spin structures correspond in the operatorial language to the two terms of the GSO projection for each of the NS-NS and RR fermions. Eventually one has to integrate over the $z, w$ vertex positions on the cylinder and over the cylinder length $l$, giving the final scattering amplitude as

$$
\mathcal{M}=\int_{0}^{\infty} d l l^{-\frac{3}{2}} e^{\frac{-Y^{2}}{2 l}} \iint d^{2} z d^{2} w A(l ; z, w) .
$$

We are interested in the case when the vertices only contain uncompactified coordinates. In principle $A(l ; z, w)$ also contains a sum over the discrete compactified momenta $p_{n}$ (they are zero if there are Neumann boundary conditions in the corresponding directions, or for Dirichlet compactified directions one has a wave function like for the uncompactified case). Since later we will be interested in the $l \rightarrow \infty$ corner of the moduli space (because it will correspond to the field theory limit) and since each momentum $p_{n}$ is weighted by a factor $e^{-l p_{n}^{2}}$ from the compact Hamiltonian, only the $p_{n}=0$ term of the sum will be relevant. In conclusion, we can take the momenta in the compact direction to be zero for both the Neumann and Dirichlet compactified directions. Correspondingly we refer to our branes as 0-branes, even when they could be Neumann in the internal compact directions.

The partition function is the result of the functional integration without vertex insertions (i.e. there is 1 in the place of the vertices). It is a product of the integration over the bosonic coordinates, the $b-c$ ghosts, the fermionic coordinates and the $\beta-\gamma$ ghosts.

The $b-c$ ghost contribution cancels the contribution of the bosonic pair $X^{0} X^{1}$. The $\beta-\gamma$ ghost contribution is like the inverse of the fermionic pair $\psi^{0} \psi^{1}$ for every spin structure, and thus it cancels this latter contribution.

Thus, the partition function has the form of the "light-cone" expression

$$
Z_{s}=Z_{L C}^{B} \cdot Z_{L C, s}^{F}
$$

where $Z_{L C}^{B}=\prod_{\mu=2,4,6,8} Z_{\mu}^{B}$ is the product of the contributions of the $X^{\mu}, X^{\mu+1}$ pairs, and $Z_{L C, s}^{F}=\prod_{\mu=2,4,6,8} Z_{\mu, s}^{F}$ is the product of the contributions of the $\psi^{\mu}, \psi^{\mu+1}$ pairs. 
With our conventions $\left\{\psi_{n}^{\mu}, \psi_{-n}^{\nu}\right\}=\eta^{\mu \nu}$, with $\eta^{00}=-1$, and $\left(\psi_{n}^{\mu}\right)^{\dagger}=\mp \psi_{-n}^{\mu}$ with upper/lower sign for $\mu=0$ or $\neq 0$. Thus $\left( \pm \psi_{n}^{0}+\psi_{-n}^{1}\right) / \sqrt{2}$ and, for $\mu \neq 0,\left(\psi_{n}^{\mu} \pm i \psi_{-n}^{\mu+1}\right) / \sqrt{2}$ are like fermionic destruction or creation operators. The contribution of each pair is derived in Appendix B.

In order to compute $\langle U V\rangle_{s}$ we need the explicit form of the correlators which are given in Section [1. Correlators can involve a pair of $X$ 's, a pair of $\psi$ 's, a pair of $\bar{\psi}$ 's, or one $\psi$ and one $\bar{\psi}$.

We show in Appendix $\mathrm{C}$ that for the particular case of the graviton-axion amplitude, which we consider in this paper, only the odd spin structure contributes. We recall that the odd spin structure corresponds to a part of the GSO projection of the RR fermions. We also anticipate that the $\sigma_{2}$ untwisted case for the compactified coordinates does not contribute for the relevant odd spin structure.

In the odd spin structure case the fermionic coordinates have zero modes and therefore the result will be zero unless the amplitude contains a sufficient number of fermionic fields. In the relevant - twisted - orbifold case the zero modes only appear for the noncompactified coordinates $\mu=0,1,2,3$. Thus, only the terms in the expression of $\langle U V\rangle$ containing a factor $\psi^{0} \psi^{1} \psi^{2} \psi^{3}$ will contribute to the path integral (remember that some $\bar{\psi}$ 's can appear in the place of $\psi$, with the identification of eq.(2.4)). We can still express the result in the form of eq. (3.4), with the understanding that the partition function contains the zero modes insertion. For this odd spin structure sector we obtain $Z_{L C}^{B} \cdot Z_{L C \text {,odd }}^{F}=1$, as in this case the part of the GSO projection of the RR fermions exactly compensates the bosonic contribution (see Appendix B).

Therefore in eq.(3.4) we need to compute the remaining part of $\langle U V\rangle_{\text {odd }}$. Altogether this amounts to saying that we compute $\left\langle U V>_{\text {odd }}\right.$ by Wick's theorem, using the correlators specified above, and factorizing in each term a factor

$$
<\psi^{\mu} \psi^{\nu} \psi^{\rho} \psi^{\sigma}>_{o d d}=-i \epsilon^{\mu \nu \rho \sigma}
$$

to keep into account the hermiticity properties and the sign of the different permutations. 
We recall again that

$$
<\psi^{\mu}\left(x_{1}\right) \psi^{\nu}\left(x_{2}\right)>_{\text {odd }}=-\eta^{\mu \nu} \partial_{x_{1}} G\left(x_{1}, x_{2}\right)
$$

and note that the nonholomorphic part of the rhs actually cancels when summing the various terms of $\left\langle U V>_{\text {odd }}\right.$.

\section{BOUNDARY STATE FOR THE RR CASE}

Here we review the construction of the boundary state for the RR case and show that the $\sigma_{2}$ untwisted odd spin structure does not contribute to our amplitude.

Consider first the four uncompactified fermionic coordinates $\psi^{\mu}$, with $\mu=0,1,2,3$. When performing the Fourier analysis in $\sigma_{2}$ we get modes $n=0$ in the RR case: they can be identified with the $\gamma$-matrices $\gamma^{\mu}=i \sqrt{2} \psi_{0}^{\mu}$ and $\tilde{\gamma}^{\mu}=i \sqrt{2} \bar{\psi}_{0}^{\mu}$, with $\left\{\gamma^{\mu}, \gamma^{\nu}\right\}=-2 \eta^{\mu \nu}$, which act on a subspace which is a direct product of two spinor spaces $S_{i} \otimes \tilde{S}_{j}$. Let us examine the sector of the boundary state related to this subspace. Since we have Neumann boundary condition in time and Dirichlet in space, we have for the boundary state $\mid B \eta>$

$$
\begin{array}{r}
\left(\gamma^{0}+i \eta \tilde{\gamma}^{0}\right) \mid B \eta>0 \\
\left(\gamma^{\mu \neq 0}-i \eta \tilde{\gamma}^{\mu \neq 0}\right) \mid B \eta>=0
\end{array}
$$

where $\eta= \pm 1$ has been put for later convenience. Let us define $a=\left(\gamma^{0}+\gamma^{1}\right) / 2$, $a^{*}=\left(\gamma^{0}-\gamma^{1}\right) / 2$ and $b=\left(-i \gamma^{2}+\gamma^{3}\right) / 2, b^{*}=\left(-i \gamma^{2}-\gamma^{3}\right) / 2$ such that $\left\{a, a^{*}\right\}=\left\{b, b^{*}\right\}=1$, similarly for $\tilde{a}, \tilde{b}$, and zero for the other anticommutators. The conditions on $\mid B \eta>$ can now be rewritten as

$$
\begin{aligned}
& \left(a+i \eta \tilde{a}^{*}\right)|B \eta>=0, \quad(b-i \eta \tilde{b})| B \eta>=0 \\
& \left(a^{*}+i \eta \tilde{a}\right)\left|B \eta>=0, \quad\left(b^{*}-i \eta \tilde{b}^{*}\right)\right| B \eta>=0
\end{aligned}
$$

Defining a "vacuum" $|0>\otimes| \tilde{0}>$ by $a|0>=b| 0>=0, \tilde{a}\left|0>=\tilde{b}^{*}\right| 0>=0$, we find the boundary state 


$$
\left|B \eta>=\frac{1}{\sqrt{2}} e^{-i \eta\left(a^{*} \tilde{a}^{*}-b^{*} \tilde{b}\right)}\right| 0>\otimes \mid \tilde{0}>
$$

which can be expressed as a sum of products of spinors

$$
\mid B \eta>=\sum_{i=1}^{4} c_{i}(\eta) S_{i} \otimes \tilde{S}_{i}
$$

One can construct explicitly the spinors and see that each $S_{i}, \tilde{S}_{i}$ is an eigenstate of $\gamma^{5}, \tilde{\gamma}^{5}$ and moreover that for each $i$ : $\gamma^{5} S_{i}=-\tilde{\gamma}^{5} \tilde{S}_{i}$.

Thus, the boundary state for the 0-brane is only compatible with Type IIA theory [4. Also, one can analyse the spacetime content of the boundary state by computing $\sum_{i} c_{i}(\eta) S_{i} C(\gamma-$ matrices $) \tilde{S}_{i}$, where $C$ is the charge conjugation matrix. It is seen that the result is different from zero only for the case of $\gamma^{0}$ and $\gamma^{5} \gamma^{0}$, consistently with the picture that the 0-brane at rest is the source for the 0-component of a vector or axialvector.

Moreover

$$
\gamma^{5}\left|B \eta>=-\tilde{\gamma}^{5}\right| B \eta>=-\mid B-\eta>
$$

The GSO projected partition function f can be written as

$$
\frac{1}{2}(<B \eta|B \eta>-<B-\eta| B \eta>) .
$$

Due to the fact that changing the sign of the $\bar{\psi}$ 's in $\mid B \eta>$ is the same as changing the sign of $\eta$, the GSO projection for the $\sigma_{2}$-Fourier $n=0$ modes amounts to the projection

$$
<B \eta\left|\frac{1+\gamma^{5}}{2} \frac{1-\tilde{\gamma}^{5}}{2}\right| B \eta>
$$

Actually $<B \eta \mid B \eta>=2$ and $<B-\eta\left|\gamma^{5}\right| B \eta>=-2$, whereas $<B-\eta \mid B \eta>=0$ which corresponds to the fact that the odd spin structure partition function is zero. The odd spin

\footnotetext{
*Recall that the $n=0$ fermionic modes do not enter into the Hamiltonian which allows us to write the partition function, eq. (3.5), in this simple symbolic form where $\mid B \eta>$ refers only to the $n=0$ modes.
} 
structure contribution is different from zero when one inserts $\psi^{\mu} \psi^{\nu} \psi^{\rho} \psi^{\sigma}$ which here is the same as $-i \epsilon^{\mu \nu \rho \sigma} \gamma^{5}$, consistently with rules for constructing the scattering amplitude in the odd spin structure case, see Section [11].

Of course, in order to construct the complete boundary state one has to include the $\sigma_{2}$-Fourier $n \neq 0$ part, and also to include the compactified coordinates part.

In general, the full partition function is

$$
Z=\frac{1}{3} \sum_{\{g\}} \sum_{\{h\}} Z(\text { total })_{g, h}
$$

where $\{g\}=1, g, g^{2}$ and $\{h\}=1, h, h^{2}$ are twists along the $\sigma_{1}$ and $\sigma_{2}$ directions for the fields corresponding to the compactified directions. Here by $Z($ total) we mean $Z($ total $)=Z^{\text {bosons }} \cdot Z^{\text {fermions }}$. Remember that one has also to project over the $Z_{3}$ invariant states, and this is implemented by the average over $g$. The compactified fermionic coordinates have zero modes in the $\sigma_{2}$-untwisted sector only. Now consider the $Z($ total $)$ just for the untwisted compactified sector and for the odd spin structure only. In this case the contributions to the partition function from the bosonic and fermionic modes for $n \neq 0$ exactly compensate leaving

$$
Z_{g, 1}^{c}=Z_{g}^{c, f e r m i o n s}(n=0)
$$

where the superscript $c$ is used to indicate the compactified part. One can now construct the action of $g$ on the boundary state in the following way. Namely, consider the $(4,5)(6,7)$ $(8,9)$ pairs, and construct for each of them the $b, b^{\dagger}$ operators like we have done for the $(2,3)$ pair. The action of an element $g$ of $Z_{3}$ amounts to $b_{(j, j+1)} \rightarrow g_{j} b_{(j, j+1)}$, with $g_{j}=e^{\frac{2 \pi i z_{j}}{3}}$ such that $z_{4}+z_{6}+z_{8}=0 \bmod 2$ [16] (see Appendix B). Denoting by $\mid B_{c} \eta>$ the boundary state for the $n=0$ modes of the compactified sector, we thus have

$$
g \cdot\left|B_{c} \eta>=\prod_{j=4,6,8} e^{i \eta g_{j}\left(b^{*} \tilde{b}\right)_{j, j+1}}\right| 0>\otimes \mid \tilde{0}>
$$

and the $Z_{3}$ invariant combination is

$$
\left|B_{c} \eta>_{i n v}=\frac{1}{3}\left(1+g+g^{2}\right)\right| B_{c} \eta>
$$


The odd spin structure contribution is found to vanish:

$$
<B_{c}-\eta \mid B_{c} \eta>_{i n v}=0
$$

because of the condition $\sum_{l} z_{l}=0 \bmod 2$. Since our vertices do not contain compactified coordinates, the $\sigma_{2}$-untwisted odd spin structure case does not contribute.

For us, therefore, the relevant case is the $\sigma_{2}$ twisted sector of the orbifold, where there are no $n=0$ modes for the compactified coordinates. Besides, as we have said in Section [II, the odd spin structure partition function with the suitable insertion of $i \psi^{0} \psi^{1} \psi^{2} \psi^{3}$ is equal to 1 , since the bosonic contribution compensates the fermionic contribution, for each higher- $n$ mode, both in the uncompactified and in the (twisted) compactified sector, and also for the ghosts. Thus, in this case we have just to compute the correlator $\left\langle U V>_{\text {odd }}\right.$, with the rules of Section III.

\section{TWO POINT FUNCTIONS ON THE CYLINDER}

Let us consider the scattering of two massless string states $\left(p^{2}=0\right)$ whose vertex operators are

$$
V(z, \bar{z})=\epsilon_{\mu \nu}\left(\partial X^{\mu}(z, \bar{z})+i p \cdot \psi(z) \psi^{\mu}(z)\right)\left(\bar{\partial} X^{\nu}(z, \bar{z})+i p \cdot \bar{\psi}(\bar{z}) \bar{\psi}^{\nu}(\bar{z})\right) e^{i p \cdot X(z, \bar{z})}
$$

with $\epsilon_{\mu \nu}$ transverse $\left(p^{\mu} \epsilon_{\mu \nu}=p^{\nu} \epsilon_{\mu \nu}=0\right)$ and symmetric for gravitons $\left(\epsilon_{\mu \nu}=\epsilon_{\nu \mu} ; \epsilon_{\mu}^{\mu}=0\right)$ and

dilatons $\left(\epsilon_{\mu \nu}=\eta_{\mu \nu}-p_{\mu} l_{\nu}-l_{\mu} p_{\nu}\right.$, where $\left.p \cdot l=1\right)$ and antisymmetric for antisymmetric tensor particles. Here $\partial \equiv \partial_{z}$ and $\bar{\partial} \equiv \partial_{\bar{z}}$. The general expression for the scattering amplitude on the cylinder has been given as equation (3.6) in Section [II].

We show in Appendix A that summing over all spin structures, amounting to the GSO projection, yields a vanishing vacuum functional at the one-loop level [4]. Recalling the analysis in Ref. [17 it is easy to show that one loop amplitudes for up to two external legs also vanish in ten dimensions.

However, when this process is considered on the orbifold the result is more interesting. Without loss of generality, we take the time as the unique Neumann direction, and the 
polarization tensors to be non-vanishing only in the directions perpendicular to the 0-brane, i.e. $\epsilon_{00}=\epsilon_{0 i}=\epsilon_{i 0}=0 ; \epsilon_{i j} \neq 0$.

We now explicitly consider the scattering of a graviton and an antisymmetric tensor, with polarization tensors $h_{i j}$ and $b_{i j}$ respectively, off the two 0-branes. The two vertex operators are, respectively,

$$
\begin{aligned}
V_{h}(k, z, \bar{z}) & =h_{i j}\left(\partial X^{i}(z, \bar{z})+i k \cdot \psi(z) \psi^{i}(z)\right)\left(\bar{\partial} X^{j}(z, \bar{z})+i k \cdot \bar{\psi}(\bar{z}) \bar{\psi}^{j}(\bar{z})\right) e^{i k \cdot X(z, \bar{z})} \\
V_{b}(p, w, \bar{w}) & =b_{l m}\left(\partial X^{l}(w, \bar{w})+i p \cdot \psi(w) \psi^{l}(w)\right)\left(\bar{\partial} X^{m}(w, \bar{w})+i p \cdot \bar{\psi}(\bar{w}) \bar{\psi}^{m}(\bar{w})\right) e^{i p \cdot X(w, \bar{w})}
\end{aligned}
$$

with

$$
\begin{array}{r}
k_{i} h_{i j}=0, \quad h_{i j}=h_{j i} \quad \sum_{i} h_{i i}=0 \\
p_{l} b_{l m}=0, \quad b_{l m}=-b_{m l} .
\end{array}
$$

We show in Appendix $\mathrm{C}$ that the contribution from the even spin structures vanishes. Let us then consider the odd spin structure sector. As we have seen in Section III a non vanishing result requires the presence of four fermionic zero modes. We denote the possible zero mode contributions as

$$
\begin{array}{r}
<\psi^{\mu} \psi^{\nu} \psi^{\rho} \psi^{\sigma}>=(-i) \epsilon^{\mu \nu \rho \sigma}, \quad<\psi^{\mu} \bar{\psi}^{\nu} \psi^{\rho} \psi^{\sigma}>=(-i) \epsilon^{\mu \tilde{\nu} \rho \sigma} \\
<\psi^{\mu} p \cdot \bar{\psi}^{\nu} \psi^{\rho} \psi^{\sigma}>=(-i) \epsilon^{\mu \nu \rho \sigma} \tilde{p}_{\nu} \text {, etc. }
\end{array}
$$

where $\tilde{\nu}$ means that one must change the sign whenever it is a space index. Similarly $\tilde{p}_{\nu}$ signifies that the space part is opposite to the space part of $p_{\nu}$.

It is convenient to summarize the odd fermionic propagators, equation (2.8), once again in this section as follows:

$$
<\psi^{\mu}(z) \psi^{\nu}(w)>=-\eta^{\mu \nu} F(z-w), \quad<\psi^{\mu}(z) \bar{\psi}^{\nu}(\bar{w})>=-\eta^{\mu \tilde{\nu}} F(z-\bar{w}),
$$

where 


$$
\begin{aligned}
& F(z-w)=\frac{\vartheta_{1}^{\prime}(z-w)}{\vartheta_{1}(z-w)}+\frac{i \pi}{l}(\operatorname{Im} z-\operatorname{Im} w) \\
& F(z-\bar{w})=\frac{\vartheta_{1}^{\prime}(z-\bar{w})}{\vartheta_{1}(z-\bar{w})}+\frac{i \pi}{l}(\operatorname{Im} z+\operatorname{Im} w)
\end{aligned}
$$

Another useful piece of notation is the contraction

$$
<k \cdot \psi(z) p \cdot \bar{\psi}(\bar{w})>=-k \cdot \tilde{p} F(z-\bar{w})
$$

where $k \cdot \tilde{p}=-\mathbf{k} \cdot \mathbf{p}-k_{0} p_{0}$.

The normal ordering of the exponential factors gives

$$
e^{-k_{\mu} p_{\nu}<X^{\mu}(z) X^{\nu}(w)>} \equiv\left|\frac{\vartheta_{1}(z-w)}{\vartheta_{1}(z-\bar{w})}\right|^{q^{2}}\left|\frac{\vartheta_{1}^{2}(z-\bar{w})}{\vartheta_{1}(z-\bar{z}) \vartheta_{1}(w-\bar{w})}\right|^{2 k_{0}^{2}} e^{\frac{2 \pi}{l} q^{2} \operatorname{Im} z \operatorname{Im} w+\frac{2 \pi k_{0}^{2}}{l}(\operatorname{Im} z-\operatorname{Im} w)^{2}}
$$

after using the following kinematical relations

$$
\begin{array}{r}
p^{0}=-p_{0}=k_{0} \\
q^{2}=(k+p)^{2}=2 k \cdot p \\
k_{i}^{2}=p_{i}^{2}=k_{0}^{2} .
\end{array}
$$

Note that the branes cannot transfer energy, but they can transfer momenta. Thus $q^{2}$ is purely spacelike $\left(q^{2}=\vec{q}^{2}\right)$.

Instead of using the antisymmetric polarization tensor, $b_{l m}$, we will write our amplitude in terms of the axion $a$ introduced as

$$
-p_{0} b_{l m}=\frac{a}{2} \epsilon_{l m s} p_{s}
$$

where $\epsilon_{l m s}$ is now the usual Levi-Civita symbol. One now does the Wick contractions, remembering that four fermions have always to be taken as zero modes to get a non-zero answer. This means that one only gets contributions when there are four, six or eight fermion fields. After some lengthy calculations we find 


$$
\begin{aligned}
<V_{h}(k, z, \bar{z}) V_{b}( & p, w, \bar{w})>=e^{-k_{\mu} p_{\nu}<X^{\mu}(z) X^{\nu}(w)>} a q \cdot h \cdot q\left[\frac { q ^ { 2 } } { 2 } \left\{-|F(z-w)|^{2}+|F(z-\bar{w})|^{2}\right.\right. \\
+ & \left.\frac{1}{2}(F(z-w))^{2}+\frac{1}{2}(F(\bar{z}-\bar{w}))^{2}-\frac{1}{2}(F(z-\bar{w}))^{2}-\frac{1}{2}(F(\bar{z}-w))^{2}\right\} \\
& -2 k_{0}^{2}\left\{|F(z-\bar{w})|^{2}-\frac{1}{2}(F(\bar{z}-w))^{2}-\frac{1}{2}(F(z-\bar{w}))^{2}\right. \\
& -F(w-\bar{w})[F(z-w)-F(z-\bar{w})-F(\bar{z}-\bar{w})+F(\bar{z}-w)] \\
+ & \frac{1}{2}[F(z-w) F(z-\bar{w})+F(\bar{z}-\bar{w}) F(\bar{z}-w) \\
& -F(z-\bar{w}) F(\bar{z}-\bar{w})-F(z-w) F(\bar{z}-w)]\} \\
& \left.-\frac{1}{2}\left(\partial_{z} \partial_{w} P_{D}+\partial_{\bar{z}} \partial_{\bar{w}} P_{D}-\partial_{z} \partial_{\bar{w}} P_{D}-\partial_{\bar{z}} \partial_{w} P_{D}\right)\right]
\end{aligned}
$$

where

$$
q \cdot h \cdot q=q^{i} h_{i j} q^{j}
$$

and

$$
P_{D}=G(z, w)-G(z, \bar{w})
$$

with $G\left(x_{1}, x_{2}\right)$ defined in eq. (2.6).

\section{PINCHING LIMIT}

In the last section we obtained the final form of the amplitude, eq. (5.11) for the scattering of a graviton and axion from two parallel 0-branes. We saw that the only contribution to this amplitude comes from the odd spin structure. Our aim in this section is to make contact with the field theory limit and to investigate the strongest singularity for $q^{2} \rightarrow 0$. This will give us the leading behaviour at large distances. We therefore analyze the behaviour of

$$
\int d^{2} z d^{2} w<V_{h}(k, z, \bar{z}) V_{b}(p, w, \bar{w})>
$$

in the limit $l \rightarrow \infty$ (field theory limit) and also $z \rightarrow w$ (pinching limit).

We first analyze the limit $z \rightarrow w$. The leading singularity in $q^{2}$ in this limit comes from the term $|F(z-w)|^{2}$. In Appendix $\mathrm{C}$ we show that this term away, from $w \sim z$, and also all the other terms in the amplitude eq. (5.11) give less singular results for $q^{2} \rightarrow 0$. 
Clearly, upon using Eq. (5.8) and the definition of $F(z-w)$, eq. (5.6), and the behaviour of $\vartheta_{1}(z-w)$, one finds that the contribution from the integration region $z \rightarrow w$ is of the form

$$
I=\int d^{2} z d^{2} w|F(z-w)|^{2} e^{-k_{\mu} p_{\nu}<X^{\mu}(z) X^{\nu}(w)>u=z-w \rightarrow 0} \longrightarrow d^{2} w \int d^{2} u \frac{|u|^{q^{2}}}{|u|^{2}}\left|\frac{\vartheta_{1}^{\prime}(0)}{\vartheta_{1}(w-\bar{w})}\right|^{q^{2}} e^{\frac{2 \pi}{l} q^{2}(\operatorname{Im} w)^{2}} .
$$

Converting to polar coordinates $u=\rho e^{i \theta}$ one can write this integral as

$$
\pi \int_{0}^{\Lambda^{2}} d \rho^{2}\left(\rho^{2}\right)^{-1+\frac{q^{2}}{2}}\left|\frac{\vartheta_{1}^{\prime}(0)}{\vartheta_{1}(w-\bar{w})}\right|^{q^{2}} e^{\frac{2 \pi}{l} q^{2}(\operatorname{Imw})^{2}}
$$

where the cutoff $\Lambda^{2}$ denotes a small integration region near $z-w=0$. One can now do the $\rho^{2}$ integration and also the integration over Rew, as the integrand is only a function of Imw, to obtain

$$
\frac{2 \pi}{q^{2}}\left(\Lambda^{q^{2}}\right) \int_{0}^{l} d \operatorname{Imw}\left|\frac{\vartheta_{1}^{\prime}(0)}{\vartheta_{1}(2 i \operatorname{Im} w)}\right|^{q^{2}} e^{\frac{2 \pi}{l} q^{2}(\operatorname{Im} w)^{2}} .
$$

Using the fact that

$$
\vartheta_{1}(z \mid 2 i l) \stackrel{l \rightarrow \infty}{\longrightarrow}-2 e^{\frac{-\pi l}{2}} \sin (\pi z)
$$

one finds

$$
\frac{\vartheta_{1}^{\prime}(0)}{\vartheta_{1}(2 i \operatorname{Im} w)} \stackrel{l \rightarrow \infty}{\longrightarrow} \frac{\pi}{\sin (2 \pi i \operatorname{Im} w)} \stackrel{\operatorname{Imw} \longrightarrow^{\infty}}{\longrightarrow}-2 \pi i e^{-2 \pi I m w}
$$

The last limit follows since we are looking for the integration region where $\operatorname{Imw} \sim l$.

Thus in this limit we find

$$
I=\frac{2 \pi}{q^{2}}(2 \pi \Lambda)^{q^{2}} \int_{0}^{l} d \operatorname{Im} w e^{-2 \pi q^{2} \operatorname{Im} w\left(1-\frac{\operatorname{Imw}}{l}\right)} .
$$

Then substituting this behaviour in eq. (3.6), and keeping all the factors from eq. (5.11), one finds that

$$
\mathcal{M} \stackrel{l \rightarrow \infty}{\longrightarrow} \frac{a}{2} q \cdot h \cdot q q^{2} \int_{0}^{\infty} d l l^{-\frac{3}{2}} I
$$


Now changing variables from $\operatorname{Im} w$ to $\eta=(\operatorname{Im} w) / l$ one finds that

$$
\begin{array}{r}
\mathcal{M} \rightarrow \pi a q \cdot h \cdot q \int_{0}^{\infty} d l l^{-1 / 2} \int_{0}^{1} d \eta e^{-2 \pi l q^{2} \eta(1-\eta)} \\
=\pi a q \cdot h \cdot q \frac{1}{\sqrt{q^{2}}} \int_{0}^{\infty} d x x^{-1 / 2} \int_{0}^{1} d \eta e^{-2 \pi x \eta(1-\eta)} \\
=a q \cdot h \cdot q \frac{1}{\sqrt{\vec{q}^{2}}} \cdot(\text { constant })
\end{array}
$$

noting that the integral over $\eta$ for large $x$ behaves as $1 / x$, and thus the subsequent integral is finite. We recall that $q^{2}$ is purely spacelike.

Since the limit $l \rightarrow \infty$ corresponds to the exchange of the lowest closed string states between the branes, the pinching limit graviton-axion amplitude has the correct momentum structure to be interpreted as the graph in figure 3, i.e the graviton and axion interact with the 0-branes through the exchange of an intermediate axion which couples to the lowest states being exchanged between the branes. In fact, the pinching limit, as usual, selects the one particle exchange in the momentum transfer channel and the obvious candidate for this particle is the axion, whose coupling to the graviton through the energy momentum tensor corresponds to the right vertex in the diagram and to the structure $a q \cdot h \cdot q$. Thus in the vertex at the left one sees the coupling of the axion with the lowest RR states exchanged between the branes. The propagators of these RR states carry only three momenta and no energy. The fact that the amplitude does not contain a pole like $1 / \vec{q}^{2}$, but only $1 / \sqrt{\vec{q}^{2}}$, suggests that the axion-RR-RR vertex is proportional to two powers of momenta. In fact, since the RR propagators behave as $1 / \vec{k}^{2}$ and $1 /(\vec{q}-\vec{k})^{2}$, integration over the $\vec{k}$, by dimensional reasons, will give $\sim \vec{q}^{2} / \sqrt{\vec{q}^{2}}$, which multiplied by the axion propagator $1 / \vec{q}^{2}$ and the right vertex $a q \cdot h \cdot q$ reproduces our result for the amplitude. 


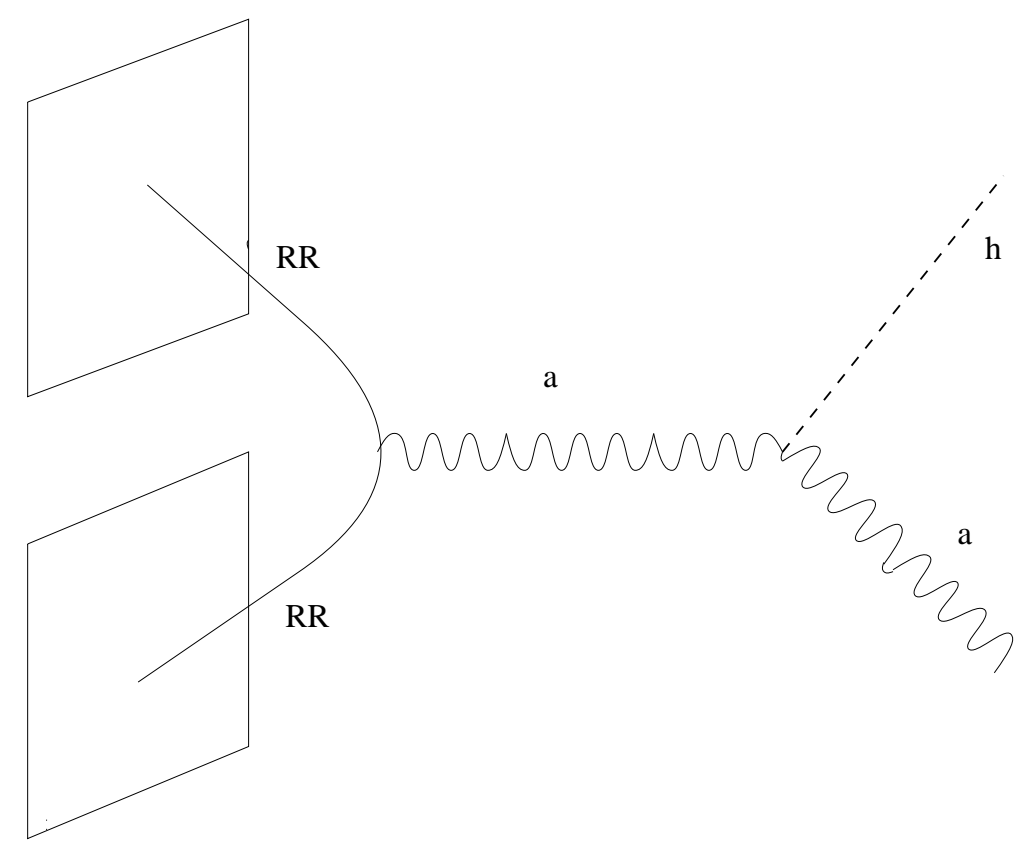

Figure 3

This interpretation of our result (let us stress that our result was obtained independently of the above field theory construction) may pose some problems. In fact it looks at variance with known rules from $N=2$ supersymmetric gauge theories, which are supposed to hold in the "bulk", away from the branes, since the axion should be in a hypermultiplet and the RR states in a vector multiplet. It would seem that the well-known breaking of $N=2$ down to $N=1$ due to the branes sort of propagates away from them. This effect could be related to the fact that the RR states coming off the branes are necessarily off-shell since they carry zero energy, whereas one can verify that the four point amplitude $R R-h \rightarrow R R-a$ vanishes (and thus also its momentum transfer pole vanishes) for on-shell RR states in agreement with the above rules.

One notices also from eq. (6.9) that, if we make a three dimensional Fourier transform of the $1 / \sqrt{\vec{q}^{2}}$ behaviour of the amplitude, we find a $1 / r^{2}$ distribution for the static axion field at large distances from the source. This is to be contrasted with the normal behaviour of a scalar field which goes like $1 / r$, and can be interpreted as due to the fact that the axion 
is coupled to a halo around the pointlike sources rather than to the sources directly.

\section{Acknowledgements.}

We would like to thank E. Gava, T. Jayaraman, K.S. Narain, A. Pasquinucci and H. Sarmadi for very useful discussions.

\section{Appendix A. D-brane vacuum amplitude}

In this Appendix we review the computation of the vacuum amplitude for two parallel branes performed by Polchinski [4] in order to introduce notation.

The mode expansion of the bosonic field $X^{\mu}\left(\sigma_{1}, \sigma_{2}\right)$ is

$$
\begin{aligned}
X^{\mu}\left(\sigma_{1}, \sigma_{2}\right) & =x^{\mu}+i k^{\mu} \sigma_{1}+\frac{i}{\sqrt{4 \pi}} \sum_{n} \frac{1}{n}\left(\alpha_{n}^{\mu} e^{2 \pi n\left(\sigma_{1}+i \sigma_{2}\right)}+\tilde{\alpha}_{n}^{\mu} e^{2 \pi n\left(\sigma_{1}-i \sigma_{2}\right)}\right) \\
& \equiv x^{\mu}+i k^{\mu} \sigma_{1}+X_{R}^{\mu}\left(\sigma_{1}, \sigma_{2}\right)+X_{L}^{\mu}\left(\sigma_{1}, \sigma_{2}\right)
\end{aligned}
$$

As is reviewed in Section [II], the bosonic boundary conditions are implemented, in the closed string formulation, by constructing a bosonic boundary state $\mid B>_{B}$. Suppose to be general we have Neumann boundary conditions for $X^{A}$ and Dirichlet for $X^{i}$. Then, if the vacuum is

defined as usual as $\alpha_{n}^{\mu}\left|0>=\tilde{\alpha}_{n}^{\mu}\right| 0>=0$, for $n>0$, it is seen that $\mid B>_{B}$ can be represented in terms of raising operators as

$$
\left|B_{B}>=\exp \left\{\sum_{n=1}^{\infty} \frac{1}{n}\left(-\eta_{A B} \alpha_{-n}^{A} \tilde{\alpha}_{-n}^{B}+\delta_{i j} \alpha_{-n}^{i} \tilde{\alpha}_{-n}^{j}\right)\right\}\right| 0>
$$

For the fermions, it is convenient to treat the NS-NS and R-R sectors separately. Let us consider the NS-NS case first. The mode expansions are

$$
\psi^{\mu}\left(\sigma_{1}, \sigma_{2}\right)=\sum_{m=-\infty}^{\infty} \psi_{m}^{\mu} e^{2 \pi m\left(\sigma_{1}+i \sigma_{2}\right)}, \quad \bar{\psi}^{\mu}\left(\sigma_{1}, \sigma_{2}\right)=\sum_{m=-\infty}^{\infty} \tilde{\psi}_{m}^{\mu} e^{2 \pi m\left(\sigma_{1}-i \sigma_{2}\right)}
$$

for $m$ half odd integer, and the boundary state is fixed by the condition (using the conventions of ref. 《4)

$$
\left(\psi_{m}^{\mu}+i \eta \tilde{\psi}_{-m}^{\mu}\right) \mid B>=0
$$


where $\eta= \pm$ is $+(-)$ for Neumann (Dirichlet) boundary conditions.

If $\psi_{m}^{\mu}\left|0>=\tilde{\psi}_{m}^{\mu}\right| 0>=0$ for half-odd $m>0$, it is seen that the boundary state satisfying condition (A.4) is

$$
\left|B, \eta>_{N S}=\exp \left\{-i \eta \sum_{m=1 / 2}^{\infty} \psi_{-m} \cdot \tilde{\psi}_{-m}\right\}\right| 0>
$$

The GSO projection is performed by the operator

$$
(-1)^{F}=-(-1)^{\sum_{m>0} \psi_{-m} \cdot \psi_{m}}
$$

which acts on $\mid B, \eta>_{N S}$ as

$$
(-1)^{F}\left|B, \eta>_{N S}=(-1)^{\tilde{F}}\right| B, \eta>_{N S}=-\mid B,-\eta>_{N S}
$$

Therefore, the GSO projected state is

$$
\mid \mathcal{B}>_{N S}=\frac{1}{2}\left\{\left|B, \eta>_{N S}-\right| B,-\eta>_{N S}\right\}
$$

¿From now on we consider the Light Cone (LC) expressions, thus keeping only the contributions of the coordinates $\mu=2, \ldots, 9$. Recalling the expression for the Hamiltonian in terms of the oscillators

$$
\begin{aligned}
H & =\frac{k^{2}}{2}+2 \pi\left\{\sum_{n \geq 1}\left(\alpha_{-n} \cdot \alpha_{n}+\tilde{\alpha}_{-n} \cdot \tilde{\alpha}_{n}\right)+\sum_{m=1 / 2}^{\infty} m\left(\psi_{-m} \cdot \psi_{m}+\tilde{\psi}_{-m} \cdot \tilde{\psi}_{m}\right)-1\right\} \\
& =\frac{k^{2}}{2}-2 \pi+H_{B}+H_{F}
\end{aligned}
$$

one can compute the NS-NS contribution to the partition function, which, after integrating over $k$, can be expressed as

$$
\begin{aligned}
Z_{N S}= & \int_{0}^{\infty} d l(2 \pi l)^{-\left(\frac{9-p}{2}\right)} e^{-\frac{Y^{2}}{2 l}} e^{2 \pi l}<B\left|e^{-l H_{B}}\right| B>_{B} \times \\
& \frac{1}{2}\left\{<B, \eta\left|e^{-l H_{F}}\right| B, \eta>_{N S}-<B, \eta\left|e^{-l H_{F}}\right| B,-\eta>_{N S}\right\} \\
= & 8 \pi^{4} V_{p+1} \int_{0}^{\infty} d l(2 \pi l)^{-\left(\frac{9-p}{2}\right)} e^{-\frac{Y^{2}}{2 l}} \vartheta_{1}^{\prime}(0 \mid 2 i l)^{-4}\left[\vartheta_{3}(0 \mid 2 i l)^{4}-\vartheta_{4}(0 \mid 2 i l)^{4}\right]
\end{aligned}
$$

where $V_{p+1}=<0 \mid 0>$ is the volume of the D-brane. 
We recall the standard definitions

$$
\begin{aligned}
& \vartheta_{2}(z \mid 2 i l)=\vartheta\left[\begin{array}{c}
1 / 2 \\
0
\end{array}\right](z \mid 2 i l) \\
& \vartheta_{3}(z \mid 2 i l)=\vartheta\left[\begin{array}{l}
0 \\
0
\end{array}\right](z \mid 2 i l) \\
& \vartheta_{4}(z \mid 2 i l)=\vartheta\left[\begin{array}{c}
0 \\
1 / 2
\end{array}\right](z \mid 2 i l) .
\end{aligned}
$$

In a similar way we can proceed in the $\mathrm{R}-\mathrm{R}$ sector. In this case, the mode expansions are the same as in eq.(A.3) but with $m$ integer. Here, the vacuum is degenerate due to the zero $\operatorname{modes} \psi_{0}^{\mu}, \tilde{\psi}_{0}^{\mu}$.

The boundary state satisfying

$$
\left(\psi_{m}^{\mu}+i \eta \tilde{\psi}_{-m}^{\mu}\right) \mid B, \eta>_{R}=0
$$

for every $m$ including $m=0$ is

$$
\left|B, \eta>_{R}=\exp \left\{-i \eta \sum_{m \geq 1} \psi_{-m} \cdot \tilde{\psi}_{-m}\right\}\right| 0, \eta>
$$

In this sector, the GSO projection is performed by the operator

$$
(-1)^{F}=\psi_{0}^{11}(-)^{\sum_{m>0} \psi_{-m} \cdot \psi_{m}}
$$

which yields

$$
\mid \mathcal{B}>_{R}=\frac{1}{2}\left(\left|B, \eta>_{R}-\right| B,-\eta>_{R}\right)
$$

It is seen that the term corresponding to the odd spin structure is $\left\langle B \eta\left|e^{-H_{F} l}\right| B-\eta\right\rangle_{R}=$ 0 (it is proportional to $\vartheta_{1}(0)^{4}=0$ ). Here $H_{F}$ is the Hamiltonian for the RR fermions. Repeating the calculation above (i.e. Eq.A.10) with the appropriate Hamiltonian) it is seen that

$$
Z_{R}=8 \pi^{4} V_{p+1} \int_{0}^{\infty}(2 \pi l)^{-\left(\frac{9-p}{2}\right)} e^{-\frac{Y^{2}}{2 l}} \vartheta_{1}^{\prime}(0 \mid 2 i l)^{-4} \vartheta_{2}(0 \mid 2 i l)^{4}
$$

In order to make the algebraic sum of the NS-NS and RR sectors, we take the same signs which hold for the partition function on the torus. Thus, the full partition function is: 


$$
Z=8 \pi^{4} V_{p+1} \int_{0}^{\infty}(2 \pi l)^{-\left(\frac{9-p}{2}\right)} e^{-\frac{Y^{2}}{2 l}} \vartheta_{1}^{\prime}(0 \mid 2 i l)^{-4}\left[\vartheta_{2}(0 \mid 2 i l)^{4}-\vartheta_{3}(0 \mid 2 i l)^{4}+\vartheta_{4}(0 \mid 2 i l)^{4}\right] .
$$

It vanishes due to the abstruse identity, reflecting the fact that there is no net force between BPS states.

\section{Appendix B. D-brane vacuum amplitude on orbifolds}

In this Appendix we consider an orbifold compactification to four dimensions. The standard $Z_{3}$ orbifold in string theory is known to break some of the supersymmetries due to the inclusion of twisted boundary conditions. Thus it is interesting to see whether the force between BPS states is affected by this more realistic setup.

Now the compactified fields are propagating along the cylinder. It is possible to construct the boundary states in this case, taking into account the twisted sectors provided by the orbifold construction. Consider the discrete group $G=Z_{3}$ that acts on the coordinates $X^{\mu}$, $\psi^{\mu}$ as $\pm 2 \pi / 3$ rotations on pairs of them. An orbifold is constructed by compactifying the coordinates $\mu=4,5,6,7,8,9$ on a 6 -torus and by identifying points that are equivalent up to an element $g$ of $G$, i.e.

$$
X^{\mu}\left(\sigma_{1}, \sigma_{2}=1\right)=g X^{\mu}\left(\sigma_{1}, \sigma_{2}=0\right)
$$

This is possible if the mode $x^{\mu}$ is a fixed point, i.e. $x^{\mu}=g x^{\mu}$. For $g \neq 1$ we call this case a twisted sector in $\sigma_{2}$.

This divides the Hilbert space into sectors where the only change is in the boundary

conditions. Moreover, since $G$ is a symmetry of the Hamiltonian, we must project onto $G$-invariant states with the projector $\sum_{k} g^{k}$, both for the twisted and untwisted sectors in $\sigma_{2}$. This projector acts similarly to the GSO projection, that is given a boundary state $\mid B>$ we have to form $\sum_{k} g^{k} \mid B>$.

In order to construct the boundary states, let us consider Dirichlet boundary conditions on the bosonic fields, i.e. $X^{\mu}\left(\sigma_{1}=0, \sigma_{2}\right)=Y_{1}^{\mu}, X^{\mu}\left(\sigma_{1}=l, \sigma_{2}\right)=Y_{2}^{\mu}$, where $Y_{1}^{\mu}$ and $Y_{2}^{\mu}$ are the positions of the branes. (If the twisted sector in $\sigma_{2}$ is considered, then $Y_{1}$ and $Y_{2}$ must be fixed points of the orbifold). These conditions can be written (in the notation of eq. (A.1. Appendix A) as 


$$
\left.\partial_{\sigma_{2}} X_{R}\right|_{\sigma_{1}=0}=-\left.\left.\partial_{\sigma_{2}} X_{L}\right|_{\sigma_{1}=0} \quad \partial_{\sigma_{2}} X_{R}\right|_{\sigma_{1}=l}=-\left.\partial_{\sigma_{2}} X_{L}\right|_{\sigma_{1}=l}
$$

Similarly for Neumann boundary conditions we have

$$
\left.\partial_{\sigma_{1}} X_{R}\right|_{\sigma_{1}=0}=-\left.\left.\partial_{\sigma_{1}} X_{L}\right|_{\sigma_{1}=0} \quad \partial_{\sigma_{1}} X_{R}\right|_{\sigma_{1}=l}=-\left.\partial_{\sigma_{1}} X_{L}\right|_{\sigma_{1}=l}
$$

In this way we can construct the states $\mid B>$ which only contain the nonzero modes. Now, for every state we have to project over the $Z_{3}$ invariant content. This is performed by applying $\sum_{m, n} g^{m} \tilde{g}^{n}$, where $g$ and $\tilde{g}$ are elements of $Z_{3}$ and act on the left and right movers respectively. This implies that the condition to be satisfied by the $Z_{3}$ projected boundary states will be deformed as

$$
\begin{array}{ll}
\left.g^{m} \partial_{\sigma_{2}} X_{L}\right|_{\sigma_{1}=0}=-\left.\tilde{g}^{n} \partial_{\sigma_{2}} X_{R}\right|_{\sigma_{1}=0} & \left.g^{m^{\prime}} \partial_{\sigma_{2}} X_{L}\right|_{\sigma_{1}=l}=-\left.\tilde{g}^{n^{\prime}} \partial_{\sigma_{2}} X_{R}\right|_{\sigma_{1}=l} \\
\left.g^{m} \partial_{\sigma_{1}} X_{L}\right|_{\sigma_{1}=0}=-\left.\tilde{g}^{n} \partial_{\sigma_{1}} X_{R}\right|_{\sigma_{1}=0} & \left.g^{m^{\prime}} \partial_{\sigma_{1}} X_{L}\right|_{\sigma_{1}=l}=-\left.\tilde{g}^{n^{\prime}} \partial_{\sigma_{1}} X_{R}\right|_{\sigma_{1}=l}
\end{array}
$$

and one has to sum over all the possibilities. Since the partition function depends only on the relative phase of $g^{m} \tilde{g}^{n}$ with respect to $g^{m^{\prime}} \tilde{g}^{n^{\prime}}$ and further of $g^{m^{\prime}}$ with respect to $\tilde{g}^{n^{\prime}}$, the Dirichlet and Neumann boundary conditions on the projected states can be summarized respectively as

$$
\begin{aligned}
& \left.\partial_{\sigma_{2}} X_{R}\right|_{\sigma_{1}=l}=-\left.g \partial_{\sigma_{2}} X_{L}\right|_{\sigma_{1}=l} \\
& \left.\partial_{\sigma_{1}} X_{R}\right|_{\sigma_{1}=l}=-\left.g \partial_{\sigma_{1}} X_{L}\right|_{\sigma_{1}=l}
\end{aligned}
$$

for the states $|B g>\equiv g| B>$ on the brane at $\sigma_{1}=l$. Thus, the operation $g$ induces a twist in the $\sigma_{1}$ direction.

Notice that, alternatively, one could interprete eq.(B.5) in the following way: due to the identifications introduced by the orbifold, it is also possible to implement the boundary conditions eqs.(B.2) or (B.3) by equating the derivative of the right movers to (minus) the derivative of the twisted left movers.

¿From now on we will consider Dirichlet boundary conditions in every space coordinate. Thus we are in the case of the D0-brane. Actually, we would get the same Light Cone 
partition function also with Neumann conditions in the compactified directions - taking the orbifold fixed points would seem anyhow to imply the Dirichlet ones; however, in the orbifold case, the integration over the compactified positions, which would be done in the Neumann case, means also a discrete sum over the fixed points. Thus when we say 0-brane, we make essentially reference to the uncompactified coordinates.

Working in light-cone coordinates $\mu, \nu=2, \ldots, 9$, let us consider the compactified directions $4, \ldots, 9$. It is convenient to introduce complex fields

$$
X^{4,5}=X^{4}+i X^{5} \quad \bar{X}^{4,5}=X^{4}-i X^{5}
$$

(and similarly $X^{6,7}, X^{8,9}$ ). The eigenvalues of $g$ acting on these complex fields are

$$
g=\exp \left\{2 \pi i\left(z_{4}+z_{6}+z_{8}\right)\right\}
$$

where $z_{4} \pm z_{6} \pm z_{8}=0 \bmod 2$. (see [16]). In order to preserve one supersymmetry, $G$ is assumed to be an abelian subgroup of $S U(3)$. Therefore, for a $Z_{3}$ orbifold, $z_{a}=n_{a} / 3$ and $n_{4} \pm n_{6} \pm n_{8}=6 n$

We first discuss the untwisted sector in $\sigma_{2}$.

Let us consider $X^{4,5}$. The corresponding oscillator modes

$$
\beta_{n}=\alpha_{n}^{4}+i \alpha_{n}^{5}, \quad \beta_{n}^{*}=\alpha_{n}^{4}-i \alpha_{n}^{5}
$$

with

$$
\left[\beta_{n}, \beta_{l}\right]=0, \quad\left[\beta_{n}, \beta_{-l}^{*}\right]=2 n \delta_{l m}
$$

and $\tilde{\beta}_{n}, \tilde{\beta}_{n}^{*}$ defined in a similar way for the left movers, yield the following condition on the boundary state

$$
\left(\beta_{n}+g_{4} \eta \tilde{\beta}_{-n}\right) \mid B_{4}, g_{4}, \eta>=0
$$

Notice that we are using $\eta$ to distinguish Neumann and Dirichlet boundary conditions. This is meant to stress the similarity between the projection over $Z_{3}$ and GSO invariant states. 
As far as we consider branes with the same Neumann and Dirichlet coordinates, the bosonic content of the bra and ket boundary states on both branes will coincide and $\eta$ will only refer to the GSO projection.

It is thus seen that

$$
\left|B_{4}, g_{4}, \eta>=\exp \left\{-\eta \sum_{n \geq 1} \frac{1}{2 n}\left(g_{4}^{*} \beta_{-n} \tilde{\beta}_{-n}^{*}+g_{4} \beta_{-n}^{*} \tilde{\beta}_{-n}\right)\right\}\right| 0>
$$

It is now possible to compute the bosonic contribution to the vacuum amplitude, taking into account the expression for the Hamiltonian in terms of the new oscillators, namely

$$
H=\pi \sum_{n}\left(\beta_{-n} \beta_{n}^{*}+\beta_{-n}^{*} \beta_{n}+\tilde{\beta}_{-n} \tilde{\beta}_{n}^{*}+\tilde{\beta}_{-n}^{*} \tilde{\beta}_{n}\right)
$$

One then gets

$$
<B_{4}, g_{4}, \eta\left|e^{-l H}\right| B_{4}, g_{4}^{\prime}, \eta>=\prod_{n=1}\left|\frac{1}{1-g_{4}^{*} g_{4}^{\prime} e^{-4 \pi l n}}\right|^{2}=\prod_{n=1}\left[1+q^{4 n}-2 \cos \left(2 \pi z_{4}\right) q^{2 n}\right]^{-1}
$$

where we have introduced $q=e^{-2 \pi l}$ and $g_{4}^{*} g_{4}^{\prime}=e^{2 \pi i z_{4}}$.

In terms of Jacobi theta functions this expression can be rewritten as

$$
<B_{4}, g_{4}, \eta\left|e^{-l H}\right| B_{4}, g_{4}^{\prime}, \eta>=\frac{2 f\left(q^{2}\right) q^{1 / 4} \sin \left(\pi z_{4}\right)}{\vartheta_{1}\left(z_{4} \mid 2 i l\right)}
$$

where

$$
f\left(q^{2}\right)=\prod_{n=1}\left(1-q^{2 n}\right)=\left(\frac{\vartheta_{1}^{\prime}(0 \mid 2 i l)}{2 \pi q^{1 / 4}}\right)^{1 / 3}
$$

Taking now into account all the contributions from the compactified directions as well as the $(2,3)$ spacetime sector and the normal ordering term in the hamiltonian $\left(q^{-2 / 3}\right)$, it is seen that the bosonic sector produces (in the notation of eq. (3.7))

$$
Z_{L C}^{B}=\left[2 f\left(q^{2}\right)\right]^{4} \frac{\pi q^{1 / 3}}{\vartheta_{1}^{\prime}(0 \mid 2 i l)} \prod_{a} \frac{\sin \left(\pi z_{a}\right)}{\vartheta_{1}\left(z_{a} \mid 2 i l\right)} .
$$

Let us now consider the NS fermionic sector. Complex fermions are defined as

$$
\chi^{(4,5)}=\psi^{4}+i \psi^{5}, \quad \chi^{(4,5) *}=\psi^{4}-i \psi^{5}
$$


(and similarly for $\chi^{(6,7)}$ and $\chi^{(8,9)}$ and the left movers $\left.\tilde{\chi}, \tilde{\chi}^{*}\right)$ with

$$
\left\{\chi_{n}, \chi_{m}\right\}=\left\{\chi_{n}, \tilde{\chi}_{m}\right\}=\left\{\tilde{\chi}_{n}, \tilde{\chi}_{m}\right\}=0 \quad\left\{\chi_{n}, \chi_{-m}^{*}\right\}=\left\{\tilde{\chi}_{n}, \tilde{\chi}_{-m}^{*}\right\}=2 \delta_{n m}
$$

The condition to be satisfied by the twisted boundary state is

$$
\left(\chi_{n}+i g \eta \tilde{\chi}_{-n}\right) \mid B, g, \eta>=0
$$

(where we avoid repeating the pairs of indices $(4,5),(6,7),(8,9)$ on the fields, the twists and the states). It is seen that the state satisfying condition $(\mathbb{B . 1 9})$ is

$$
\left|B, g, \eta>=\exp \left\{-\frac{\eta i}{2} \sum_{n}\left(g \chi_{-n}^{*} \tilde{\chi}_{-n}+g^{*} \chi_{-n} \tilde{\chi}_{-n}^{*}\right)\right\}\right| 0>\text {. }
$$

Taking into account all the compactified directions as well as the $(2,3)$ spacetime sector, the full LC boundary state is

$$
|\mathcal{B}>=| B, \eta>_{23}\left|B, g_{4}, \eta_{4}>\right| B, g_{6}, \eta_{6}>\mid B, g_{8}, \eta_{8}>
$$

Now the vacuum amplitude can be computed, using the expression for the Hamiltonian in terms of the complex fields,

$$
H=\pi \sum_{m=1 / 2}^{\infty} m\left(\chi_{-m} \chi_{m}^{*}+\chi_{-m}^{*} \chi_{m}+\tilde{\chi}_{-m} \tilde{\chi}_{m}^{*}+\tilde{\chi}_{-m}^{*} \tilde{\chi}_{m}\right)
$$

Proceeding similarly as in the bosonic case, we find (with $g^{*} g^{\prime}=e^{2 \pi i z_{a}}$ )

$$
\begin{aligned}
& <B, g, \eta\left|e^{-l H}\right| B, g^{\prime}, \eta^{\prime}>= \\
& =\prod_{n}\left|1+\eta \eta^{\prime} g^{*} g^{\prime} e^{-4 \pi l n}\right|=\prod_{n=1}^{\infty}\left|1 \pm e^{2 \pi i z_{a}} q^{(2 n-1)}\right|^{2}
\end{aligned}
$$

(Note that $\eta \eta^{\prime}= \pm$ for the two possible cases of the GSO projection). In order to compute the partition function we have to put together all the compactified directions as well as the spacetime contribution, perform the GSO projection and include the normal ordering term in the hamiltonian $\left(q^{-1 / 3}\right)$. Finally the contribution in the NS sector amounts to, in the notation of eq. (3.7),

$$
Z_{L C\left(N S \eta \eta^{\prime}=1\right)}^{F}-Z_{L C\left(N S \eta \eta^{\prime}=-1\right)}^{F}=\frac{\vartheta_{3}(0 \mid 2 i l) \prod_{a} \vartheta_{3}\left(z_{a} \mid 2 i l\right)}{q^{1 / 3}\left[f\left(q^{2}\right)\right]^{4}}-\frac{\vartheta_{4}(0 \mid 2 i l) \prod_{a} \vartheta_{4}\left(z_{a} \mid 2 i l\right)}{q^{1 / 3}\left[f\left(q^{2}\right)\right]^{4}} .
$$


Let us now turn our attention to the $\mathrm{R}$ sector. In this case the $n=0$ modes deserve a special treatment, which we have discussed in Section $\mathbb{I \nabla}$. We recall from that Section that the $n=0$ contribution in the odd spin structure case gives a vanishing result after the projection $\sum_{k} g^{k} \mid B>$ is taken into account. Instead, the $n=0$ mode part for the even spin structure case does not vanish. Rather, it gives a factor $\prod_{a} 2 \cos \left(\pi k z_{a}\right)$ for each $g^{k}, k \neq 0$.

The $n \neq 0$ contribution in this sector, for a pair of compactified coordinates, amounts to

$$
\begin{array}{r}
<B\left|e^{-l H}\right| g^{k} B>_{\eta \eta^{\prime}=1}=\prod_{n=1}^{\infty}\left|1+e^{2 \pi i z_{a}} q^{2 n}\right|^{2} \\
=\prod_{n=1}^{\infty}\left[1+q^{4 n}+2 q^{2 n} \cos \left(2 \pi z_{a}\right)\right]
\end{array}
$$

which in terms of Jacobi theta functions is

$$
\frac{\vartheta_{2}\left(z_{a} \mid 2 i l\right)}{2 f\left(q^{2}\right) q^{1 / 4} \cos \left(\pi z_{a}\right)}
$$

Putting together the contributions from the $(2,3)$ spacetime fermions and the internal $(4,5),(6,7),(8,9)$ directions, after including the zero point energy factor $q^{2 / 3}$ we get

$$
Z_{L C\left(R \eta \eta^{\prime}=1\right)}^{F}=\frac{\vartheta_{2}(0 \mid 2 i l) \prod_{a} \vartheta_{2}\left(z_{a} \mid 2 i l\right)}{q^{1 / 3} f\left(q^{2}\right)^{4}}
$$

In order to make the algebraic sum of the R and NS sectors, we take the same signs which hold for the partition function on the torus. Thus we get the $L C$ partition function

$$
\begin{aligned}
& Z_{L C}=Z_{L C}^{B} \cdot\left(Z_{L C\left(R \eta \eta^{\prime}=1\right)}^{F}-Z_{L C\left(N S \eta \eta^{\prime}=1\right)}^{F}+Z_{L C\left(N S \eta \eta^{\prime}=-1\right)}^{F}\right)= \\
& \frac{16 \pi}{\vartheta_{1}^{\prime}(0 \mid 2 i l)} \prod_{a} \frac{\sin \left(\pi z_{a}\right)}{\vartheta_{1}\left(z_{a} \mid 2 i l\right)} \\
& \times\left\{\vartheta_{2}(0 \mid 2 i l) \prod_{a} \vartheta_{2}\left(z_{a} \mid 2 i l\right)-\vartheta_{3}(0 \mid 2 i l) \prod_{a} \vartheta_{3}\left(z_{a} \mid 2 i l\right)+\vartheta_{4}(0 \mid 2 i l) \prod_{a} \vartheta_{4}\left(z_{a} \mid 2 i l\right)\right\} .
\end{aligned}
$$

which vanishes due to the Riemann identity.

When the position of the brane is on the fixed point of the orbifold one has also to include the twisted sector in $\sigma_{2}$. In this case, the fields in the compactified dimensions may be diagonalized such that

$$
X^{a, b}\left(\sigma_{2}+1\right)=e^{2 \pi i z_{a}} X^{a, b}\left(\sigma_{2}\right), \quad X^{* a, b}\left(\sigma_{2}+1\right)=e^{-2 \pi i z_{a}} X^{* a, b}\left(\sigma_{2}\right)
$$


Therefore, the mode expansion of $X^{a, b}$ and $X^{* a, b}$ is given by

$$
\begin{gathered}
X^{a, b}=x^{a b}+\frac{i}{\sqrt{4 \pi}} \sum_{n \in Z}\left[\frac{1}{\sqrt{n-1 / 3}} \gamma_{n} e^{-2 \pi i(n-1 / 3) \sigma_{2}}+\frac{1}{\sqrt{n-2 / 3}} \tilde{\gamma}_{n} e^{2 \pi i(n-2 / 3) \sigma_{2}}\right] \\
X^{* a, b}=x^{* a b}+\frac{i}{\sqrt{4 \pi}} \sum_{n \in Z}\left[\frac{1}{\sqrt{n-2 / 3}} \gamma_{n}^{*} e^{-2 \pi i(n-2 / 3) \sigma_{2}}+\frac{1}{\sqrt{n-1 / 3}} \tilde{\gamma}_{n}^{*} e^{2 \pi i(n-1 / 3) \sigma_{2}}\right]
\end{gathered}
$$

where

$$
\gamma_{n}\left|0>=0, \quad \tilde{\gamma}_{n}\right| 0>=0, \quad \gamma_{n}^{*}\left|0>=0, \quad \tilde{\gamma}_{n}^{*}\right| 0>=0
$$

for $n>0$. Therefore $\gamma_{n}^{\dagger}=\gamma_{-n+1}^{*}$ and $\tilde{\gamma}_{n}^{\dagger}=\tilde{\gamma}_{-n+1}^{*}$ and the commutation relations are

$$
\left[\gamma_{n}, \gamma_{-l+1}^{*}\right]=2 \delta_{n l}, \quad n, l>0, \quad\left[\gamma_{l+1}, \gamma_{n}^{*}\right]=2 \delta_{n l}, \quad n, l \leq 0
$$

In terms of the new oscillators, the Hamiltonian is

$$
H=\pi \sum_{n=0}\left[\left(n+\frac{1}{3}\right)\left(\gamma_{-n} \gamma_{n+1}^{*}+\tilde{\gamma}_{-n}^{*} \tilde{\gamma}_{n+1}\right)+\left(n+\frac{2}{3}\right)\left(\gamma_{-n}^{*} \gamma_{n+1}+\tilde{\gamma}_{-n} \tilde{\gamma}_{n+1}^{*}\right)\right]
$$

and the condition to be verified by the boundary state is

$$
\left(\gamma_{n}+\eta g \tilde{\gamma}_{-n+1}\right) \mid B>=0
$$

It is easy to see that the boundary state verifying it is

$$
\left|B>=\exp \left\{-\frac{\eta}{2} \sum_{n=1}\left(g \gamma_{-n+1}^{*} \tilde{\gamma}_{-n+1}+g^{*} \gamma_{-n+1} \tilde{\gamma}_{-n+1}^{*}\right)\right\}\right| 0>
$$

Therefore, the contribution from this sector to the partition function is

$$
\begin{aligned}
& <B, g, \eta\left|e^{-l H}\right| B, g^{\prime}, \eta>= \\
& \prod_{n=1}\left(1-g^{*} g^{\prime} e^{-4 \pi l\left(n-\frac{1}{3}\right)}\right)^{-1}\left(1-g g^{\prime *} e^{-4 \pi l\left(n-\frac{2}{3}\right)}\right)^{-1}
\end{aligned}
$$

with $\left(g^{*} g^{\prime}=e^{2 \pi i z_{a}}\right)$.

After putting all the contributions from the spacetime and internal directions together, the full bosonic part of the path integral is in the notation of eq.(3.7)

$$
Z_{L C h}^{B}=\prod_{a} \prod_{n=1}\left[1-e^{2 \pi i z_{a}} e^{-4 \pi l\left(n-\frac{1}{3}\right)}\right]^{-1}\left[1-e^{-2 \pi i z_{a}} e^{-4 \pi l\left(n-\frac{2}{3}\right)}\right]^{-1}
$$


where the subscript $h$ means twist in the $\sigma_{2}$ direction.

Using the infinite product expansion of the generalized $\vartheta$-functions,

$$
\begin{array}{r}
\vartheta\left[\begin{array}{l}
a \\
b
\end{array}\right](z \mid 2 i l)=q^{a^{2}} e^{2 \pi i a(b+z)} \prod_{n}\left(1-q^{2 n}\right) \prod_{n}\left(1+q^{2 n-1} e^{2 \pi i(2 i a l+b+z)}\right) \\
\prod_{n}\left(1+q^{2 n-1} e^{-2 \pi i(2 i a l+b+z)}\right)
\end{array}
$$

$Z_{L C h}^{B}$ can be expressed as

$$
Z_{L C h}^{B}=e^{\pi i / 2} f\left(q^{2}\right) q^{1 / 12}\left[\prod_{a} \vartheta\left[\begin{array}{l}
1 / 6 \\
1 / 2
\end{array}\right]\left(z_{a} \mid 2 i l\right)\right]^{-1}
$$

In order to discuss the fermionic contribution, let us start with the NS sector. The condition $(\overline{B .19})$ is now modified to

$$
\left(\chi_{n}+i g \eta \tilde{\chi}_{-n+1}\right) \mid B, g, \eta>=0
$$

which is satisfied by

$$
\left|B, g, \eta>=\exp \left\{-\frac{i \eta}{2} \sum_{n=1}\left(g \chi_{-n+1}^{*} \tilde{\chi}_{-n+1}+g^{*} \chi_{-n+1} \tilde{\chi}_{-n+1}^{*}\right)\right\}\right| 0>
$$

The corresponding Hamiltonian is

$$
H=\pi \sum_{n=0}\left[\left(n+\frac{5}{6}\right)\left(\chi_{-n} \chi_{-n+1}^{*}+\tilde{\chi}_{-n}^{*} \tilde{\chi}_{-n+1}\right)+\left(n+\frac{1}{6}\right)\left(\chi_{-n}^{*} \chi_{-n+1}+\tilde{\chi}_{-n} \tilde{\chi}_{-n+1}\right)\right]
$$

and therefore, each complex pair of directions contributes

$$
Z_{L C h\left(N S \eta \eta^{\prime}\right)}^{F} \equiv<B, g, \eta\left|e^{-l H}\right| B, g^{\prime}, \eta^{\prime}>=\prod_{n=1}\left[1+\eta \eta^{\prime} g^{*} g^{\prime} e^{-4 \pi l\left(n-\frac{5}{6}\right)}\right]\left[1+\eta \eta^{\prime} g g^{\prime *} e^{-4 \pi l\left(n-\frac{1}{6}\right)}\right]
$$

Taking into account the spacetime and the three pairs of complex directions, and using Eq.(B.37), the full contribution of the NS sector can be written as

$$
\begin{aligned}
& Z_{L C h\left(N S \eta \eta^{\prime}=1\right)}^{F}+Z_{L C h\left(N S \eta \eta^{\prime}=-1\right)}^{F}= \\
& q^{-1 / 3} f\left(q^{2}\right)^{-4}\left\{\vartheta\left[\begin{array}{l}
0 \\
0
\end{array}\right](0 \mid 2 i l) \prod_{a} \vartheta\left[\begin{array}{c}
-1 / 3 \\
0
\end{array}\right]\left(z_{a} \mid 2 i l\right)-\vartheta\left[\begin{array}{c}
0 \\
1 / 2
\end{array}\right](0) \prod_{a} \vartheta\left[\begin{array}{c}
-1 / 3 \\
1 / 2
\end{array}\right]\left(z_{a} \mid 2 i l\right)\right\} \\
& =f\left(q^{2}\right)^{-4}\left\{\vartheta\left[\begin{array}{l}
0 \\
0
\end{array}\right](0 \mid 2 i l) \prod_{a} \vartheta\left[\begin{array}{l}
0 \\
0
\end{array}\right]\left(z_{a}-2 i l / 3 \mid 2 i l\right)+\vartheta\left[\begin{array}{c}
0 \\
1 / 2
\end{array}\right](0) \prod_{a} \vartheta\left[\begin{array}{c}
0 \\
1 / 2
\end{array}\right]\left(z_{a}-2 i l / 3 \mid 2 i l\right)\right\} .
\end{aligned}
$$


Recall that in the twisted sector for the $Z_{3}$ orbifold there has to be a relative positive sign between the $\eta \eta^{\prime}=1$ and the $\eta \eta^{\prime}=-1$ sectors because of invariance under $\tau \rightarrow \tau+3$.

Let us now consider the $\mathrm{R}$ sector. In the $\sigma_{2}$ twisted sector there are no $n=0$ modes in the compactified dimensions. In order to deal with the $n=0$ modes of the uncompactified coordinates we put for them a small twist in the $\sigma_{1}$ direction, i.e. $g^{*} g^{\prime}=e^{2 \pi i \epsilon}$, otherwise the odd spin structure case $\eta \eta^{\prime}=-1$ would be identically zero. Thus we write for the $(2,3)$ spacetime directions

$$
<B, \eta\left|e^{-l H_{0}}\right| B, \eta^{\prime}>_{(2,3)}=\left(1+\eta \eta^{\prime} e^{2 \pi i \epsilon}\right) \prod_{n=1}\left|1+\eta \eta^{\prime} e^{-4 \pi l n}\right|^{2}
$$

In the case of $\eta \eta^{\prime}=1$ (even spin structure) we can directly take the limit $\epsilon \rightarrow 0$

$$
<B, \eta\left|e^{-l H_{0}}\right| B, \eta>_{(2,3)}=\frac{\vartheta_{2}(0)}{q^{1 / 4} f\left(q^{2}\right)}
$$

and thus in the notation of eq. (3.7)

$$
\begin{aligned}
Z_{L C h\left(R \eta \eta^{\prime}=1\right)}^{F} & =\frac{\vartheta_{2}(0)}{q^{1 / 4} f\left(q^{2}\right)} \prod_{a} \prod_{n=1}\left(1+e^{2 \pi i z_{a}} e^{-4 \pi l\left(n-\frac{1}{3}\right)}\right)\left(1+e^{-2 \pi i z_{a}} e^{-4 \pi l\left(n-\frac{2}{3}\right)}\right) \\
& =\frac{\vartheta\left[\begin{array}{c}
1 / 2 \\
0
\end{array}\right](0 \mid 2 i l)}{q^{1 / 3} f\left(q^{2}\right)^{4}} \prod_{a} \vartheta\left[\begin{array}{c}
1 / 6 \\
0
\end{array}\right]\left(z_{a} \mid 2 i l\right) \\
& =\frac{\vartheta\left[\begin{array}{c}
1 / 2 \\
0
\end{array}\right](0 \mid 2 i l)}{f\left(q^{2}\right)^{4}} \prod_{a} \vartheta\left[\begin{array}{c}
1 / 2 \\
0
\end{array}\right]\left(z_{a}-2 i l / 3 \mid 2 i l\right) .
\end{aligned}
$$

In the odd spin structure case $\eta \eta^{\prime}=-1$ we have for small $\epsilon$

$$
<B, \eta\left|e^{-l H_{0}}\right| B, \eta^{\prime}>_{(2,3)} \rightarrow \frac{i \epsilon \vartheta_{1}^{\prime}(0)}{q^{1 / 4} f\left(q^{2}\right)}
$$

In this case the $n \neq 0$ modes contribute the inverse of the bosonic sector (compare eq.(B.14) for $\left.z_{4} \rightarrow \epsilon\right)$, and thus we have in the notation of eq. (3.7)

$$
Z_{L C}^{B} \cdot Z_{L C\left(R \eta \eta^{\prime}=-1\right)}^{F}=-2 \pi i \epsilon \sim-2 \pi i \frac{\vartheta_{1}(\epsilon)}{\vartheta_{1}^{\prime}(0)}
$$

which is zero for $\epsilon \rightarrow 0$. But of course, as we have seen in Section [II], the insertion of vertices in the amplitude can give a nonzero result also for the odd spin structure, and we are indeed interested in this case. To be precise, one should go beyond the light cone and 
include also the $(0,1)$ and $(\beta \gamma)$ ghost contribution, with their zero modes. Altogether this gives the rules at the end of Section III (formally, the insertion of $\psi^{2} \psi^{3}$,for instance, would provide a factor proportional to $\frac{\vartheta_{1}^{\prime}(0)}{\vartheta_{1}(\epsilon)}$ to multiply eq. (B.48)).

We conclude the computation of the $L C$ partition function by assembling its even spin structure part. We take the standard combination, see eq.(43),

$$
Z_{L C h}=Z_{L C h}^{B} \cdot\left(Z_{L C h\left(N S \eta \eta^{\prime}=1\right)}^{F}+Z_{L C h\left(N S \eta \eta^{\prime}=-1\right)}^{F}-Z_{L C h\left(R \eta \eta^{\prime}=1\right)}^{F}\right)
$$

We can now show that this vanishes for each twisted sector. We see from eqs. (B.43) and (B.46) that the above equation is proportional to the sum

$$
\begin{array}{r}
\vartheta\left[\begin{array}{l}
0 \\
0
\end{array}\right](0 \mid 2 i l) \prod_{a} \vartheta\left[\begin{array}{l}
0 \\
0
\end{array}\right]\left(z_{a}-2 i l / 3 \mid 2 i l\right)+\vartheta\left[\begin{array}{c}
0 \\
1 / 2
\end{array}\right](0) \prod_{a} \vartheta\left[\begin{array}{c}
0 \\
1 / 2
\end{array}\right]\left(z_{a}-2 i l / 3 \mid 2 i l\right) \\
-\vartheta\left[\begin{array}{c}
1 / 2 \\
0
\end{array}\right](0 \mid 2 i l) \prod_{z_{a}} \vartheta\left[\begin{array}{c}
1 / 2 \\
0
\end{array}\right]\left(z_{a}-2 i l / 3 \mid 2 i l\right) .
\end{array}
$$

Writing $w_{1}=z_{4}-2 i l / 3, w_{2}=z_{6}-2 i l / 3, w_{3}=z_{8}+4 i l / 3$ one can convert the above expression to

$$
\begin{array}{r}
e^{2 \pi i\left(z_{8}+i l / 3\right)}\left\{\vartheta\left[\begin{array}{l}
0 \\
0
\end{array}\right](0 \mid 2 i l) \prod_{a} \vartheta\left[\begin{array}{l}
0 \\
0
\end{array}\right]\left(w_{a} \mid 2 i l\right)-\vartheta\left[\begin{array}{c}
0 \\
1 / 2
\end{array}\right](0) \prod_{a} \vartheta\left[\begin{array}{c}
0 \\
1 / 2
\end{array}\right]\left(w_{a} \mid 2 i l\right)\right. \\
\left.-\vartheta\left[\begin{array}{c}
1 / 2 \\
0
\end{array}\right](0 \mid 2 i l) \prod_{a} \vartheta\left[\begin{array}{c}
1 / 2 \\
0
\end{array}\right]\left(w_{a} \mid 2 i l\right)\right\}
\end{array}
$$

which vanishes due to the Riemann identity as $\sum_{a} w_{a}=0 \bmod 2$.

Finally, let us make a comment on the boundary conditions. In the case of Neumann boundary conditions in the compactified directions, we have to integrate over the branes' position and this includes a sum over the orbifold fixed points. Thus, it is like taking Dirichlet conditions on those fixed points. In the case we start by considering Dirichlet conditions in the compactified directions, we should take the branes at rest at a generic fixed position and work out the dynamics of the closed string. But we have further to consider that in the low energy state of the brane the wave function will be spread over the compactified directions. Thus we have to do an average over the compactified position of the brane, as it would be done for the nuclei wave function in a molecule within the Born Oppenheimer 
approximation. Like above, the integration over the position includes also a sum over the fixed points. In conclusion, the fixed points always contribute, bringing in the $\sigma_{2}$-twisted sector.

\section{Appendix C. Even spin structures contribution to the axion-graviton scat-}

\section{tering}

In this appendix we discuss the contribution from the even spin structures to the gravitonaxion scattering amplitude. The only possible non vanishing invariant in this case is $(p \cdot h \cdot b \cdot k)$. This can arise either from purely bosonic contractions or from terms containing four or eight fermions. Actually the contribution from the eight fermionic contractions is found to vanish. The result is

$$
\begin{array}{r}
(p \cdot h \cdot b \cdot k) \times e^{-<k \cdot X(z) p \cdot X(w)>}\left\{\partial_{z} \partial_{w} P_{D} \bar{\partial}_{z} P_{D} \bar{\partial}_{w} P_{D}-\bar{\partial}_{z} \bar{\partial}_{w} P_{D} \partial_{z} P_{D} \partial_{w} P_{D}-\partial_{z} \bar{\partial}_{w} P_{D} \bar{\partial}_{z} P_{D} \partial_{w} P_{D}\right. \\
+\bar{\partial}_{z} \partial_{w} P_{D} \partial_{z} P_{D} \bar{\partial}_{w} P_{D}+\partial_{z} \partial_{w} P_{D} F(\bar{z}-\bar{w})_{s}^{2}-\bar{\partial}_{z} \bar{\partial}_{w} P_{D} F(z-w)_{s}^{2} \\
-\partial_{z} \bar{\partial}_{w} P_{D} F(\bar{z}-w)_{s}^{2}+\bar{\partial}_{z} \partial_{w} P_{D} F(z-\bar{w})_{s}^{2}+\partial_{z} P_{D} \partial_{w} P_{D}(k \cdot p) F(\bar{z}-\bar{w})_{s}^{2} \\
\left.-\bar{\partial}_{z} P_{D} \bar{\partial}_{w} P_{D}(k \cdot p) F(z-w)_{s}^{2}-\partial_{z} P_{D} \bar{\partial}_{w} P_{D}(\tilde{k} \cdot p) F(\bar{z}-w)_{s}^{2}+\bar{\partial}_{z} P_{D} \partial_{w} P_{D}(\tilde{k} \cdot p) F(z-\bar{w})_{s}^{2}\right\}
\end{array}
$$

Here $F\left(x_{1}-x_{2}\right)_{s}$ are any of the three even spin structure fermionic propagators. As we see, the terms come out in pairs, always in the form: a term minus its complex conjugate. Now, it is easy to see that the integration over $d^{2} z d^{2} w$ produces a real result, and thus the entire contribution from the even spin structure vanishes.

Indeed, first observe that $e^{-<k \cdot X(z) p \cdot X(w)>}$ is real, then also that each term in the above expression, call it $T(z, w)$, is even by doing simultaneously $z \rightarrow-z, \bar{z} \rightarrow-\bar{z}, w \rightarrow-w, \bar{w} \rightarrow$ $-\bar{w}$. Thus

$$
\int_{-1 / 2}^{1 / 2} d \operatorname{Re} z \int_{-1 / 2}^{1 / 2} d \operatorname{Rew} T(z, w)=\int_{-1 / 2}^{1 / 2} d \operatorname{Re} z \int_{-1 / 2}^{1 / 2} d \operatorname{RewT}(\bar{z}, \bar{w})
$$

i.e. the result is real.

Also notice that the first four terms do not contain any fermionic propagators and vanish in any case because of the sum over spin structures. 


\section{Appendix D. Analysis of the field theory limit}

In this Appendix we analyze the small $q^{2}$ limit of the amplitude, arguing that the leading behaviour comes from the pinching limit $z \sim w$ of the term proportional to $|F(z-w)|^{2}$ in eq. (5.11). This leading result, which provides the strongest singularity for $q^{2} \rightarrow 0$ and thus the leading behaviour at large distances, has been analyzed in Section VI. Here we look for the $q^{2} \rightarrow 0$ limit of every term of eq. (5.11), including $|F(z-w)|^{2}$ without taking the pinching limit. The leading behaviour comes from the region of the moduli space $l \rightarrow \infty$ and $\operatorname{Imz}, \operatorname{Im} w \sim l$.

First we analyze the terms of the form $F\left(x_{1}-x_{2}\right) F\left(x_{1}^{\prime}-x_{2}^{\prime}\right)$, leaving aside those of the form $\partial_{x_{1}} \partial_{x_{2}} P_{D}$ for a later discussion.

Let us define $\zeta=\frac{I m z}{l}$ and $\eta=\frac{I m w}{l}$ and use the following asymptotic behaviour for $l \rightarrow \infty$

$$
\frac{1}{\pi} F(z-w) \rightarrow \frac{\cos \pi(z-w)}{\sin \pi(z-w)}+i(\zeta-\eta)
$$

Thus, the limit $l \rightarrow \infty$ at fixed $\zeta, \eta$ of the different terms appearing in the amplitude is

$$
\begin{array}{r}
\frac{1}{\pi} F(z-w) \rightarrow i[-\epsilon(\zeta-\eta)+\zeta-\eta] \\
\frac{1}{\pi} F(\bar{z}-\bar{w}) \rightarrow-i[-\epsilon(\zeta-\eta)+\zeta-\eta] \\
\frac{1}{\pi} F(z-\bar{w}) \rightarrow i[-1+\zeta+\eta] \\
\frac{1}{\pi} F(\bar{z}-w) \rightarrow i[-1+\zeta+\eta] \\
\frac{1}{\pi} F(w-\bar{w}) \rightarrow i[-1+2 \eta]
\end{array}
$$

(where $\epsilon( \pm x)= \pm 1$ ) and moreover

$$
\begin{aligned}
e^{-<k \cdot X(z) p \cdot X(w)>} \rightarrow & e^{-2 \pi q^{2} l \eta(1-\zeta)+2 \pi k_{0}^{2} l(\eta-\zeta)^{2}} \text { for } \zeta>\eta \\
& e^{-2 \pi q^{2} l \zeta(1-\eta)+2 \pi k_{0}^{2} l(\eta-\zeta)^{2}} \text { for } \eta>\zeta
\end{aligned}
$$

In every term we first do the integration on the difference $(\eta-\zeta)$ taking advantage of the gaussian factor $e^{2 \pi k_{0}^{2} l(\eta-\zeta)^{2}}$, interpreting it as the analytic continuation from $k_{0}^{2}<0$, obtaining a factor $\frac{1}{\sqrt{-k_{0}^{2}} \sqrt{l}}$ times the rest of the integrand for $\eta=\zeta$. 
We have then for the terms proportional to $a q^{2}(q \cdot h \cdot q)$ in eq. (5.11) the integral (keeping the large $l$ contribution)

$$
\int d l l^{-3 / 2} \frac{l^{2}}{\sqrt{-k_{0}^{2}} \sqrt{l}} \int_{0}^{1} d \zeta \zeta(1-\zeta) \cdot e^{-2 \pi q^{2} l \zeta(1-\zeta)}
$$

both for $\zeta>\eta$ and for $\eta>\zeta$. From this last integral we get a behaviour $1 / q^{2} \sqrt{-k_{0}^{2}}$, which gives for the amplitude $a q \cdot h \cdot q / \sqrt{-k_{0}^{2}}$, i.e. subleading with respect to the behaviour coming from the pinching limit.

Let us consider next the terms in eq. (5.11) proportional to $a q \cdot h \cdot q k_{0}^{2}$. It is seen from the above equations on the $l \rightarrow \infty$ behaviour that altogether they give an integrand of the form

$$
f(\zeta, \eta) \cdot e^{-<k \cdot X(z) p \cdot X(w)>}
$$

where $f(\zeta=\eta)=0$. From that one can expect, as it is confirmed by a more detailed analysis, that the subsequent integrations will give a result for the amplitude not worse than $a q \cdot h \cdot q \log \left(q^{2}\right) / \sqrt{-k_{0}^{2}}$ for $q^{2} \rightarrow 0$.

Notice that actually the set of terms

$$
-F(z-\bar{w}) F(\bar{z}-\bar{w})-F(z-w) F(\bar{z}-w)+F(z-w) F(z-\bar{w})+F(\bar{z}-\bar{w}) F(\bar{z}-w)
$$

vanishes upon the symmetric integration over $d^{2} z d^{2} w$.

Finally let us consider the terms of the kind $\partial_{x_{1}} \partial_{x_{2}} P_{D}$, where $x_{1}$ can be $z$ or $\bar{z}$ etc. Consider for instance the term $x_{1}=z, x_{2}=w$ (the discussion for the others being similar). Its behaviour for $l \rightarrow \infty$ is

$$
\sim \frac{1}{\sin ^{2} \pi(z-w)}+\frac{1}{l}
$$

We then take $z=\operatorname{Re} z+i l \zeta, w=R e w+i l \eta$ and the asymptotic behaviour of $1 / \sin ^{2}$ gives a vanishing contribution due to the integration over dRezdRew. There remains the term $\frac{1}{l} e^{-<k \cdot X(z) p \cdot X(w)>}$, which, after the gaussian integration described above and the subsequent integrations, gives for the amplitude again a result like $a q \cdot h \cdot q \log \left(q^{2}\right) / \sqrt{-k_{0}^{2}}$ for $q^{2} \rightarrow 0$. 


\section{REFERENCES}

[1] C.M. Hull and P.K. Townsend, Nucl. Phys. B438 (1995) 109;

P.K. Townsend, Phys. Lett. B350 (1995) 184

[2] E. Witten, Nucl. Phys. B443 (1995) 85

[3] M.J. Duff, "Supermembranes", hep-th/9611203

[4] J. Polchinski, Phys. Rev Lett. 75 (1995) 4724

[5] C. Callan and J. Maldacena, "D-brane approach to black hole quantum mechanics", hep -th/9602043;

G. Horowitz and A. Strominger, "Counting states of near extremal black holes", hepth/9602051;

S. Gubser, I. Klebanov and A. Peet, "Entropy and temperature of black 3-branes", hepth/9602135;

G. Horowitz, J. Maldacena and A. Strominger, "Non-extremal black holes, microstates and U-duality", hep-th/9603109

[6] S. H. Shenker, "Another length scale in String Theory?", hep-th/9509132

[7] D. Kabat and P. Pouliot, "A Comment on Zero-Brane Quantum Mechanics", hepth/9603127;

U. H. Danielsson, G. Ferretti and B. Sundborg, "D-Particle Dynamics and Bound States", hep-th/9603081

[8] I. Klebanov and L. Thorlacius, "The size of p-branes", hep-th/9510200

[9] S.S.Gubser, A. Hashimoto, I. Klebanov and J. Maldacena, "Gravitational lensing by p-branes", hep-th/9601057

[10] J. Barbón, "D-brane form factors at high energy", hep-th/9601098

[11] R. Myers and M.R.Garousi, "Superstring scattering from D-branes", hep-th/9603194 
[12] A.Hashimoto and I. Klebanov, "Decay of excited D-branes", hep-th/9604065

[13] M.J. Duff, Phys. Rev. D5 (1972) 2419

[14] C. Burgess and T.Morris, Nucl. Phys. B291 (1987) 256 and Nucl. Phys. B291 (1987) 285

[15] J. Polchinski and Y. Cai, Nucl. Phys. B296 (1988) 91

[16] J. A. Minahan, Nucl. Phys. B298 (1988) 36

[17] A. Namazie, K. Narain and H. Sarmadi, Phys. Lett. B177 (1986) 329 